\title{
Contenidos, estructura y determinantes de las ideas de los padres. Una investigación empírica
}

\section{Jesús Palacios Universidad de Sevilla*}

Las múltiples investigaciones cuyas aportaciones hemos compendiado en la revisión de literatura precedente nos han permitido aprender mucho en poco tiempo sobre las ideas que los padres tienen en relación con el desarrollo y la educación de sus hijos.

Sin embargo, nos parece que la mayor parte de las investigaciones sobre este tema han sido frecuentemente fragmentarias, pues han analizado separadamente los diversos contenidos y cualidades de las ideas de los padres, sin hacer un esfuerzo por incluirlos a todos en una misma investigación. Creemos, así mismo, que las diferencias o semejanzas entre los cónyuges han sido poco exploradas. No ha habido, a nuestro entender, suficiente esfuerzo para discernir el papel que juegan las distintas variables socio-demográficas (nivel profesional, nivel de escolarización, experiencia previa como padres, hábitat rural o urbano) sobre las ideas de los padres. Más allá de las diferencias que habitualmente suelen estudiarse entre aspectos concretos de la ideas, ha habido pocos esfuerzos por integrar en conceptualizaciones globales el conjunto de ideas que un padre

* Dirección del autor: Departamento de Psicología Evolutiva y de la Educación. Universidad de Sevilla. Apartado 3128. 41071 SEVILLA.

Agradecimientos

La realización de esta investigación se ha visto facilitada por diversas ayudas. Unas han sido de tipo material, como la beca del Comité Conjunto Hispano-Norteamericano para la Cooperación Cultural y Educativa (gracias a la que pasé una temporada en la Universidad de Yale tecopilando información y discutiendo con otros colegas), y la del Instituto de Desarrollo Regional de la Universidad de Sevilla (que me hizo posible hacer frente a los aspectos menos atractivos de la investigación empírica, relacionados todos ellos con cuestiones financieras).

Las ayudas que más agradezco son las recibidas por parte de las personas que me facilitaron las cosas en el Hospital Maternal (los doctores Ignacio Gómez de Terreros, Enrique Laza y Fernando Soler, las enfermeras Inmaculada Castro y Ana García, y la asistente social Margarita Sánchez), me ayudaron con las entrevistas o la codificación de las respuestas (Vicente Cardenal, Rosario Cubero, $M^{2}$ del Mar González, Alfonso Luque, Antonio Molina, $\mathbf{M}^{2}$ Carmen Moreno, $\mathrm{M}^{2}$ Carmen Orúe, Francisco Pleguezuelo, Anal del Valle e Isabel Viñuelas), o me asesoraron en los análisis de datos (Juan Manuel Batista, Carlos Camacho, Charo Martínez Arias y Alfonso Palmer). $\mathrm{M}^{2}$ Carmen Moreno y $\mathrm{M}^{2}$ Rosario Valdecantos leyeron el informe in extenso de la investigación y me hicieron útiles sugerencias. A todos ellos mi más profundo agradecimiento. 
o una madre sostienen, comparando entonces esa conceptualización global con la correspondiente a otro padre $\mathbf{u}$ otra madre.

Todas éstas son las cosas que hemos pretendido abordar en nuestra investigación, como primer eslabón de una cadena en la que se habrá de incluir el estudio a lo largo del tiempo de las ideas, su relación con la acción y con el desarrollo de los niños (ver en este mismo dossier, el artículo de Palacios, González y Moreno).

\section{METODO}

\subsection{Sujetos}

Hemos estudiado a un total de 278 padres (139 parejas) de entre 25 y 30 años, procedentes de distintos hábitats (rural y urbano), con distinto grado de experiencia previa como padres (parejas primíparas, segundíparas y tercíparas) y con distinto nivel de escolarización (bajo, medio y alto, según tengăn, respectivamente, sólo estudios primarios, estudios secundarios o estudios universitarios). La distribución de los efectivos de la muestra se recoge en el Cuadro 1, que contiene el número de parejas en cada una de las categorías. Naturalmente, las parejas coinciden en hábitat y paridad (hemos tenido un solo caso en que uno de los cónyuges aportó al matrimonio algún hijo habido previamente), pero no tienen por qué coincidir en nivel de estudios. Puesto que para hacer una primera composición de la muestra necesitábamos tomar a la pareja como criterio, optamos por asignar a cada pareja el nivel de estudios del padre (en el $96 \%$ de los casos, igual o superior al de su esposa). Para los análisis posteriores se tomará en consideración, lógicamente, el nivel de estudios de cada sujeto. No obstante, puesto que codificamos también el nivel de estudios de la pareja, no extrañe al lector encontrar luego en algunas muestras de ediciones de ordenador la categoría snivel de estudios de la parejas.

Una vez construida la muestra con estos criterios, aprovechamos otras informaciones socio-demográficas de los padres: profesión, experiencia previa con hermanos propios más pequeños, edad del hijo mayor (si lo tienen), etc.

\section{Cundro I}

Distribución por parejas de los efectivos de la muestra en función de las variables nivel de estudios, paridad y häbitat.

\begin{tabular}{|c|c|c|c|c|}
\hline & \multirow{2}{*}{\multicolumn{2}{|c|}{ PAR I D D }} & \multicolumn{2}{|c|}{ HABITAT } \\
\hline & & & \multirow{2}{*}{$\begin{array}{r}\text { Rural } \\
14 \\
7 \\
8\end{array}$} & \multirow{2}{*}{$\begin{array}{c}\text { Urbanc } \\
12 \\
7 \\
4\end{array}$} \\
\hline \multirow{4}{*}{ 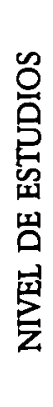 } & 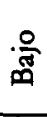 & $\begin{array}{l}\text { Primíparas } \\
\text { Segundiparas } \\
\text { Tercíparas }\end{array}$ & & \\
\hline & 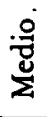 & $\begin{array}{l}\text { Primíparas } \\
\text { Segundiparas } \\
\text { Tercíparas }\end{array}$ & $\begin{array}{r}12 \\
9 \\
3\end{array}$ & $\begin{array}{r}12 \\
12 \\
1\end{array}$ \\
\hline & $\frac{8}{4}$ & $\begin{array}{l}\text { Primíparas } \\
\text { Segundiparas } \\
\text { Tercíparas }\end{array}$ & $\begin{array}{l}4 \\
5 \\
1\end{array}$ & $\begin{array}{r}15 \\
10 \\
3\end{array}$ \\
\hline & & Totales & 63 & 76 \\
\hline
\end{tabular}




\subsection{Materiales}

Para estudiar las ideas evolutivo-educativas de los padres nos hemos servido de un Cuestionario sobre ideas de los padres (CIP) elaborado por nosotros para esta investigación. El CIP consta de 106 preguntas abiertas destinadas a explorar once ámbitos diferentes. A continuación aparece la identificación de cada ámbito, el número de preguntas que se le dedica en el CIP y un ejemplo representativo del tipo de preguntas utilizadas.

- Información sobre embarazo, crianza y educación (cantidad de información que creen tener, de dónde procede, qué más les gustaría saber) (9 preguntas): «Si usted tiene alguna vez un problema o una duda importante en relación con la educación de sus hijos, ¿a quién consultaría?».

- Capacidades atribuidas al feto, al bebé y al niño (15 preguntas): «¿A qué edad cree usted que los niños pueden controlar el pipí y la caca?».

- Herencia-medio en personalidad, lenguaje e inteligencia ( 9 preguntas): « ¿A qué cree usted que se debe el hecho de que entre los niños normales unos sean más inteligentes que otros?».

- Relación madre-hijo durante el embarazo (5 preguntas): «QQué cosas cree usted que es bueno que haga una mujer embarazada porque pueden ser beneficiosas para el niño?».

- El papel del padre (4 preguntas): ${ }^{\circ}$ Cuál debe ser, a su juicio, el papel del padre en el primer año de la vida del niño?».

- Prácticas de crianza y educación (29 preguntas): «Qué cree usted que haría si ve que su hijo o hija de 4 años está jugando con sus genitales?».

- Prácticas de estimulación cognitivo-lingüísticas (6 preguntas): «Qué cosas pueden hacer los padres para favorecer la inteligencia de sus hijos?» (esta pregunta sólo se formula si, en respuesta a una pregunta anterior, los padres han admitido que se puede estimular la inteligencia).

- Atribuciones al comportamiento (8 preguntas): « Por qué cree usted que los niños pequeños se llevan todo a la boca?».

- Valores, aspiraciones y expectativas (9 preguntas): « Cómo le gustaría que fuera su hijo? ¿Qué cualidades le gustaría que tuviera? ¿Qué es lo que más desearía para él?».

- Ideas sobre la escolarización (6 preguntas): «Le parece bien que los niños vayan a guarderías?».

- Ideas sobre la constelación familiar ( 6 preguntas): «乙Cree usted que ser hijo único es bueno o malo?».

\subsection{Procedimiento}

Los padres fueron entrevistados en el Hospital Maternal de la Seguridad Social de Sevilla. Se trata de un Hospital público al que acuden a dar a luz mujeres de la ciudad de Sevilla y de su entorno. Una vez que la mujer ingresaba en el Hospital para dar a luz, comprobábamos si se ajustaba a nuestros criterios (vivir con el padre del niño, estar entre 25 y 30 años, etc.). Una vez nacido el niño visitábamos a la madre, le explicábamos sumariamente nuestro interés por lo que los padres piensan y, si los dos cónyuges estaban de acuerdo, fijábamos una cita para la entrevista ese mismo día o al día siguiente, aún dentro del Hospital.

Las entrevistas se realizaban simultáneamente al hombre y a la mujer en habitaciones contiguas pero independientes. La madre era entrevistada por una mujer y el padre por un hombre. Los entrevistadores habían sido entrenados previamente. Después de cada una de las 106 preguntas, en el CIP aparecían una 
serie de alternativas de respuesta que un estudio piloto previo había revelado como las más frecuentes. Los padres no conocían estas alternativas, sino sólo la pregunta. Si la respuesta se ajustaba a una de las alternativas previstas, el entrevistador rodeaba ésta con un círculo; si no, la respuesta se transcribía directamente debajo de la pregunta correspondiente.

\section{RESUITADOS}

Una vez codificadas las respuestas de los padres y suprimidas para el análisis algunas preguntas a las que la inmensa mayoría de los sujetos contestaban con idéntica respuesta, formamos una matriz de 278 filas (los sujetos) y 99 columnas (las preguntas del CIP y las variables sociodemográficas). Los datos de esta matriz se sometieron a dos tipos de análisis: en primer lugar, a un análisis en base a $\mathrm{X}^{2}$; en segundo lugar, a un análisis de correspondencias mứltiples, seguido de un análisis de clusters.

\subsection{Análisis en base a $\mathbf{X}^{2}$}

Por lo que al análisis en base a $\mathrm{X}^{2}$ se refiere, articularemos la exposición en torno a las principales variables socio-demográficas (sexo, experiencia previa, nivel de estudios...), pues hacerlo en torno a los diferentes bloques de contenido alargaría enormemente la exposición.

Al cruzar la variable sexo con las respuestas dadas a las preguntas del CIP, mujeres y hombres han resultado, en conjunto, más semejantes que diferentes entre sí. Algunas de las diferencias encontradas se refieren a la información sobre embarazo, crianza y educación (las mujeres realizan actividades de búsqueda de información que requieren más esfuerzos que las de los hombres) y a las prácticas de crianza y educación (las madres presentan a la vez una mayor sensibilidad respecto a las necesidades del niño y unas prácticas de socialización algo más coercitivas que las de los hombres). En otros ámbitos, como el de las predicciones respecto al calendario evolutivo, hombres y mujeres no difieren cuando se trata de los hitos más populares (edades en que se empieza a ver, oír, andar, hablar), pero sí lo hacen al predecir hechos menos conocidos [edad del control de esfínteres, cuándo los niños son capaces de hacerse entender por extraños; así, por ejemplo, las mujeres predicen una mayor precocidad que los hombres en el calendario de control de esfínteres: $\left.\mathrm{X}^{2}(4)=17.15, p=0.002\right]$.

La experiencia previa como padres no ha dado lugar a diferencias significativas importantes. Si este dato es en sí mismo interesante, más aun lo es el hecho de que las diferencias que aparecen son con mucha frecuencia inconsistentes, pues hay aspectos en que las personas primíparas difieren de las no primíparas, mientras que en otros, primíparas y tercíparas son más semejantes entre sí de lo que lo son segundíparas y tercíparas, mientras que aun en otros casos las semejanzas más acusadas se dan entre primíparas y segundíparas. Cuando se toman en consideración simultáneamente el sexo y la experiencia previa, las diferencias son también poco claras y la inconsistencia vuelve a ser un hecho frecuente.

La toma en consideración del hábitat rural o urbano de los padres de la muestra ha dado lugar a diferencias significativas en algunos ámbitos, mientras que en otros, tales diferencias no han aparecido. En conjunto, las respuestas han resultado ser significativamente diferentes en la información sobre embarazo, crianza y educación (los padres de procedencia rural se han informado más que los urbanos a través de conversaciones con otras personas, mientras que los urbanos se refieren más que los rurales a lecturas y a su propia formación como fuentes 
de información), en el papel atribuido a la herencia y el medio en la determinación de características psicológicas (los hombres y mujeres rurales resultan ser más innatistas que los urbanos, que son más ambientalistas), en las ideas sobre la relación madre-hijo durante el embarazo (los sujetos urbanos parecen mostrar una mayor sensibilidad que los rurales a las cualidades de la relación psicológica madre-hijo durante el embarazo) y en las ideas sobre prácticas de crianza y educación (la flexibilidad y permisividad de los urbanos contrastan con los estereotipos y la coercitividad de los rurales). Como ilustración de estas diferencias, el Cuadro 2 muestra los porcentajes de respuestas en la pregunta sobre la actitud a seguir ante el juego con los genitales por parte del niño $\left[\mathrm{X}^{2}(S)=36.31, p<0.001\right]$.

CuAdro 2

Diferencias entre rurales $y$ urbanos ante el juego infantil con los genitales

\begin{tabular}{|l|c|c|c|c|c|c|c|}
\hline & $\begin{array}{c}\text { Nada } \\
\text { especial }\end{array}$ & $\begin{array}{c}\text { Reñir, } \\
\text { castigar }\end{array}$ & $\begin{array}{c}\text { Dar expli- } \\
\text { caciones }\end{array}$ & $\begin{array}{c}\text { Distraer } \\
\text { al niño }\end{array}$ & $\begin{array}{c}\text { Consultar } \\
\text { especialista }\end{array}$ & No sabe & \\
\hline Rurales & 30.15 & 28.57 & 25.39 & 3.96 & 3.17 & 8.73 & 100 \\
\hline Urbanos & 57.89 & 5.92 & 26.31 & 1.97 & 3.28 & 4.60 & 100 \\
\hline
\end{tabular}

Ha sido la variable nivel de estudios la que, sin lugar a dudas, ha dado lugar a un mayor número de diferencias significativas; casi el $60 \%$ de los cruces entre esta variable y las preguntas del CIP han dado lugar a tales diferencias. La variable nivel profesional, por su parte, ha producido también importantes diferencias (un $40 \%$ de los cruces, aproximadamente), aunque menos numerosas y conceptualmente menos claras a veces que las derivadas de la variable nivel de estudios. Esta última ha dado lugar a las siguientes diferencias, algunas de las cuales ilustramos con los porcentajes de respuestas correspondientes:

- el nivel de información sobre embarazo, crianza y educación de los niños aumenta según lo hace el nivel educativo de los padres;

- los padres de nivel educativo alto suelen atribuir capacidades más precoces al feto, el bebé y el niño; los de nivel educativo bajo presentan expectativas que implican una menor precocidad; por su parte, los padres de nivel educativo medio presentan expectativas más variables, a veces intermedias entre los dos anteriores y a veces más próximos a las de unos u otros;

- los padres de nivel educativo bajo son en general más innatistas que los de nivel educativo alto, que tienden a adoptar con mayor frecuencia posiciones interaccionistas entre la herencia y la educación; los padres de nivel medio son más inestables, pues aparecen en algunas preguntas como innatistas, en otras como ambientalistas y en otras aun en una posición intermedia. El Cuadro 3 recoge los porcentajes de respuestas al preguntar por qué unos niños son más inteligentes que otros $\left[\mathrm{X}^{2}(6)=65.13, p<0.001\right]$;

\section{Cundro 3}

Diferencias entre padres de distinto nivel de estudios respecto a las causas de las diferencias interindividuales en inteligencia.

\begin{tabular}{|l|c|c|c|c|c|}
\hline & Herencia & Educación & $\begin{array}{c}\text { Interacción } \\
\text { herenc.-educ. }\end{array}$ & No sabe & \\
\hline Bajo & 43.18 & 13.63 & 9.09 & 34.09 & 100 \\
\hline Medio & 50.00 & 17.14 & 14.28 & 18.57 & 100 \\
\hline Alto & 20.83 & 16.66 & 52.77 & 9.72 & 100 \\
\hline
\end{tabular}


- los padres de nivel de estudios alto muestran una sensibilidad netamente superior a la de los otros dos grupos respecto a las cualidades psicológicas de la relación madre-niño durante el embarazo;

- las personas de nivel educativo alto y medio asignan al padre un papel más próximo a la madre durante el embarazo; respecto al papel del padre en la educación temprana del niño, los que abogan por una menor implicación son los padres de nivel educativo bajo, defendiendo los de nivel alto una elevada implicación y situándose en una posición intermedia los padres de nivel medio de escolarización;

- respecto a las prácticas de crianza y educación, los padres de elevado nivel de estudios aparecen como más razonadores e interactivos con sus hijos, se perciben como más capaces de influir sobre los niños, son más permisivos y parecen más proclives a modular su conducta en función de las circunstancias; los padres de nivel bajo parecen más orientados a la formación de hábitos, se ven a sí mismos como poco influyentes sobre algunas de las cualidades psicológicas de sus hijos y resultan ser más estereotipados y coercitivos; los padres de nivel medio ocupan en general una posición intermedia entre los dos anteriores;

- a medida que aumenta el nivel educativo de los padres, éstos se ven crecientemente capaces de influir sobre las cualidades cognitivo-lingüísticas de sus hijos y de hacerlo desde edades más tempranas. El Cuadro 4 recoge los porcentajes de respuesta obtenidos al preguntar a los padres si creen que pueden influir sobre la inteligencia de sus hijos $\left[\mathrm{X}^{2}(4)=14.97, p<0.001\right]$;

Cundro 4

Porcentajes de respuesta en función del nivel de estudios, obtenidos al preguntar a los padres sobre su capacidad para influir sobre la inteligencia de sus bijos

\begin{tabular}{|l|c|c|c|c|}
\hline & Sí & No & No sabe & \\
\hline Bajo & 64.17 & 28.35 & 7.46 & 100 \\
\hline Medio & 70.83 & 25.00 & 4.16 & 100 \\
\hline Alto & 88.88 & 9.72 & 1.38 & 100 \\
\hline
\end{tabular}

- los padres de nivel educativo alto defienden, más que los de niveles bajo y medio, valores que enfatizan la independencia de los hijos y que son menos estereotipados en función del sexo; los padres de nivel educativo alto presentan reducidas discrepancias entre sus aspiraciones y sus expectativas en relación con el futuro de sus hijos, mientras que los padres de nivel bajo son los que presentan discrepancias más elevadas; del futuro de sus hijos, a los padres de niveles bajo y medio les preocupa sobre todo la situación social general, mientras que los padres de nivel alto dicen estar más preocupados por el bienestar y la felicidad de sus hijos;

- a medida que aumenta el nivel educativo de los padres crece su convicción en el carácter positivo de la escolarización temprana, especialmente por referencia a las influencias beneficiosas de cara al desarrollo y la socialización;

- finalmente, en lo relativo a la constelación familiar, la diferencia más clara entre los padres de distinto nivel educativo radica en la disminución de ideas estereotipadas sobre el hijo único a medida que aumenta el nivel educativo; cuanto más elevado sea éste, más importancia se da a la educación que el niño reciba y menos al hecho de que tenga o no hermanos. Los porcentajes de respuesta concretos se recogen en el Cuadro $5\left[\mathrm{X}^{2}(-6)=18.26, p=0.006\right]$. 
Ideas de los padres de distinto nivel educativo referidas a si ser bijo ünico es bueno o malo.

\begin{tabular}{|l|c|c|c|c|c|}
\hline & Bueno & Malo & Depende & No sabe & \\
\hline Bajo & 2.98 & 79.10 & 15.67 & 2.23 & 100 \\
\hline Medio & .00 & 68.05 & 30.55 & 1.38 & 100 \\
\hline Alto & 1.38 & 55.55 & 40.27 & 2.77 & 100 \\
\hline
\end{tabular}

Los cruces combinados entre variables han mostrado que el nivel de estudios es determinante tanto en el caso de los hombres como en el de la mujeres, si bien las diferencias entre hombres y mujeres parecen ser más importantes entre los padres de nivel educativo bajo de lo que lo son entre los padres de niveles medio y alto. La experiencia previa como padres parece más influyente también en el caso de los padres de nivel bajo y el hábitat parece más importante entre los padres de los niveles bajo y alto de escolarización; no obstante, en estos casos la magnitud de las diferencias es escasa y no se debe sobredimensionar.

El resto de las variables socio-demográficas consideradas (experiencia previa con hermanos menores, sexo del recién nacido, edad del hijo mayor...) no ha dado lugar a diferencias significativas dignas de mención.

\subsection{Análisis de correspondencias múltiples y de «clusters»}

El análisis de correspondencias múltiples (Lebart y Morineau, 1984; Lebart, Morineau y Fenelon, 1982) nos permite un logro de gran interés en una investigación como la nuestra: descubrir las dimensiones principales de una realidad compleja y representar esas dimensiones sobre unos ejes en los que se ordenan sus elementos. La utilización posterior del análisis de clusters permite identificar clases de sujetos sobre la base de las dimensiones previamente establecidas por el análisis de correspondencias; sabremos así cómo se agrupan nuestros sujetos en clases y cómo se definen tales clases por su posición sobre los ejes ya conocidos (ver el artículo de Batista y Surela que cierra este dossier).

Por lo que a la determinación de las dimensiones se refiere, el análisis de correspondencias múltiples pone de manifiesto la existencia en nuestros datos de cuatro ejes o factores fundamentales, de los que nos proporciona las 30 modalidades más importantes para su interpretación. En la página que sigue reproducimos la edición de ordenador que contiene las 30 modalidades del primer factor, modalidades que, como se ve, aparecen ordenadas por encima y por debajo de la zona central y cuyos valores-tests van decreciendo por arriba y por abajo según se va de los extremos hacia la zona central.

Por lo que al factor 1 se refiere (Cuadro 6), nos parece el más general, el que engloba una mayor diversidad de contenidos: nivel de estudios de los padres, grado de información, expectativas evolutivas, actitudes educativas (permisividadcoerción), grado de influencia percibida, estereotipos educativos, papel asignado a lo innato y lo aprendido. Como se ve en el Cuadro 6, el factor está enmarcado por los niveles de estudios alto y bajo como modalidades de respuesta con mayor peso, situándose luego el resto de las modalidades características de este eje, que no es preciso reiterar aquí por aparecer con claridad en el Cuadro, pero que delinean con bastante precisión dos conjuntos de ideas evolutivoeducativas claramente diferenciadas: en un caso, el encabezado por el nivel de estudios alto, encontramós ideas que enfatizan la interacción entre lo heredado y lo adquirido, buen nivel de información, actitudes permisivas, expectativas 
que implican precocidad, elevado grado de influencia percibida, ideas poco estereotipadas; en el otro, el encabezado por el nivel de estudios bajo, hallamos ideas que representan la antítesis de las que acabamos de enunciar.

\section{Cundro 6}

Modalidades de respuesta que ayudan a interpretar el factor 1.

\begin{tabular}{|c|c|c|}
\hline $\begin{aligned} 1 & \text { NEP1. NEPP } \\
2 & \text { NES1. NESS } \\
3 & \text { JUG2 } \text {. JUGE } \\
4 & \text { IAN1. IANT } \\
5 & \text { INE2. INEM } \\
6 & \text { QLE6 . QLEC } \\
7 & \text { EERS. EERR } \\
8 & \text { APL3. APLE } \\
9 & \text { LEC2. LECT } \\
10 & \text { LEP2. LEPM } \\
11 & \text { PQL1. PQLE } \\
12 & \text { CIIS. CIIN } \\
13 & \text { ANT1. ANTE } \\
14 & \text { IT12. ITIM } \\
15 & \text { FAI2. FAIN }\end{aligned}$ & 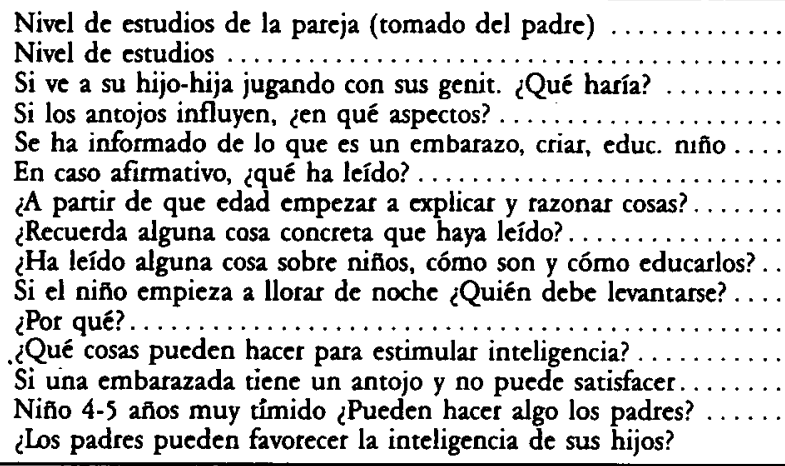 & $\begin{array}{l}\text { Bajo } \\
\text { Bajo } \\
\text { Reñir, castigar } \\
\text { Físicos } \\
\text { No } \\
\text { NO cONTESTA } \\
\text { Más de } 4 \text { años } \\
\text { NO CONTESTA } \\
\text { No } \\
\text { Madre } \\
\text { Madre sabe tratar } \\
\text { NO CONTESTA } \\
\text { Sí } \\
\text { No } \\
\text { No }\end{array}$ \\
\hline $\begin{array}{l}640 \text { PER3. PERE } \\
641 \text { EER1. EERR } \\
642 \text { APL1. APLE } \\
643 \text { LEC1. LECT } \\
644 \text { FEP4. FEPE } \\
645 \text { BEN . BENE } \\
646 \text { PAP1. PAPA } \\
647 \text { ORI4. ORID } \\
648 \text { DIT4. DITE } \\
649 \text { JUG1. JUGE } \\
650 \text { DPB3. DPBE } \\
651 \text { DIN3. DINT } \\
652 \text { PRW7. PROF } \\
653 \text { NEP3. NEPP } \\
654 \text { NES3. NESS }\end{array}$ & 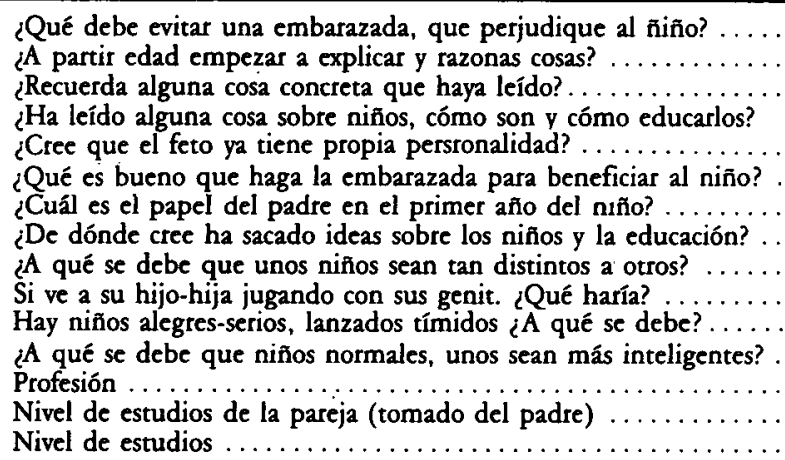 & $\begin{array}{l}\text { Esfuerzos y tensiones } \\
\text { Desde el principio } \\
\text { Cosas concretas } \\
\text { Sí } \\
\text { Sólo en parte } \\
\text { Cuidados físico-psic. } \\
\text { Igual a la madre } \\
\text { Combinaciones } \\
\text { Herencia-medio } \\
\text { Nada especial } \\
\text { Herencia-educación } \\
\text { Herencia-educación } \\
\text { Técnicos superiores } \\
\text { Alto } \\
\text { Alto }\end{array}$ \\
\hline
\end{tabular}

En resumen, este primer factor parece asociado a lo que, siguiendo a Schaefer y Edgerton (1985), podríamos denominar amodernidado de las ideas de los padres. El segundo factor nos parece referido a la influenciabilidad del desarroIlo. El tercero tiene que ver con la diferenciación de los roles sexuales. El cuarto, que resulta menos fácilmente interpretable, se relaciona con la información sobre embarazo, educación y desarrollo (con modalidades de respuesta que indican niveles medio-bajos), con una concepción ambientalista del desarrollo que paradójicamente se acompaña de un cierto pesimismo en cuanto a la posibilidad de influir sobre él y con unas expectativas evolutivas medio-bajas.

El paso siguiente en nuestro programa de análisis consiste en servirse de técnicas de clasificación automática para agrupar a individuos semejantes. El proceso se lleva a cabo con un algoritmo de clasificación de tipo jerárquico cuya lógica es la siguiente: en un primer momento, se constituyen grupos de individuos en función de las semejanzas que existen entre ellos (en base a sus características socio-demográficas y a sus respuestas a las preguntas del CIP); con los dos grupos más semejantes se forma una agregación que los contiene; a esta agregación se superpone luego la formada entre ella y el nuevo grupo de sujetos que más se le asemeje. Se van asi constituyendo y agregando grupos; las agregaciones se hacen a una distancia menor o mayor en función de la mayor o menor semejanza, respectivamente, que exista entre la agregación anterior y la nueva; los últimos grupos que se agregan son los más distantes o heterogéneos entre sí. 
En el caso de nuestros datos, este procedimiento ha desvelado la existencia de seis clases de sujetos y una séptima residual. El Cuadro 7 recoge los efectivos y el porcentaje acumulado correspondientes a cada clase. Como se re, las más importantes son las tres primeras clases de sujetos, que agrupan a casi el $90 \%$ de los efectivos de la muestra. A estas tres clases ceñiremos nuestros comentarios aquí.

\section{CuAdro 7}

Clases de sujetos, efectivos y porcentajes acumulados

\begin{tabular}{|l|c|c|c|c|c|c|c|}
\hline \multicolumn{1}{c|}{} & \multicolumn{7}{c|}{ C L A S E S } \\
\cline { 2 - 9 } & 1 & 2 & 3 & 4 & 5 & 6 & 7 \\
\hline $\mathrm{N}^{\circ}$ sujetos de la clase & 94 & 79 & 67 & 15 & 10 & 9 & 4 \\
\hline $\begin{array}{l}\text { Porcentaje acumulado } \\
\text { de individuos }\end{array}$ & 33.8 & 62.2 & 86.3 & 91.7 & 95.3 & 98.6 & 100 \\
\hline
\end{tabular}

Además, como muestra el dendrograma de la Figura 1, que representa las sucesivas agregaciones entre las clases, la 1 , la 2 y la 3 son las que mejor diferencian a unos sujetos de otros, pues las clases más distintas entre sí son, como hemos dicho antes, aquellas cuya agregación se efectúa más tardíamente.

FIGURA 1

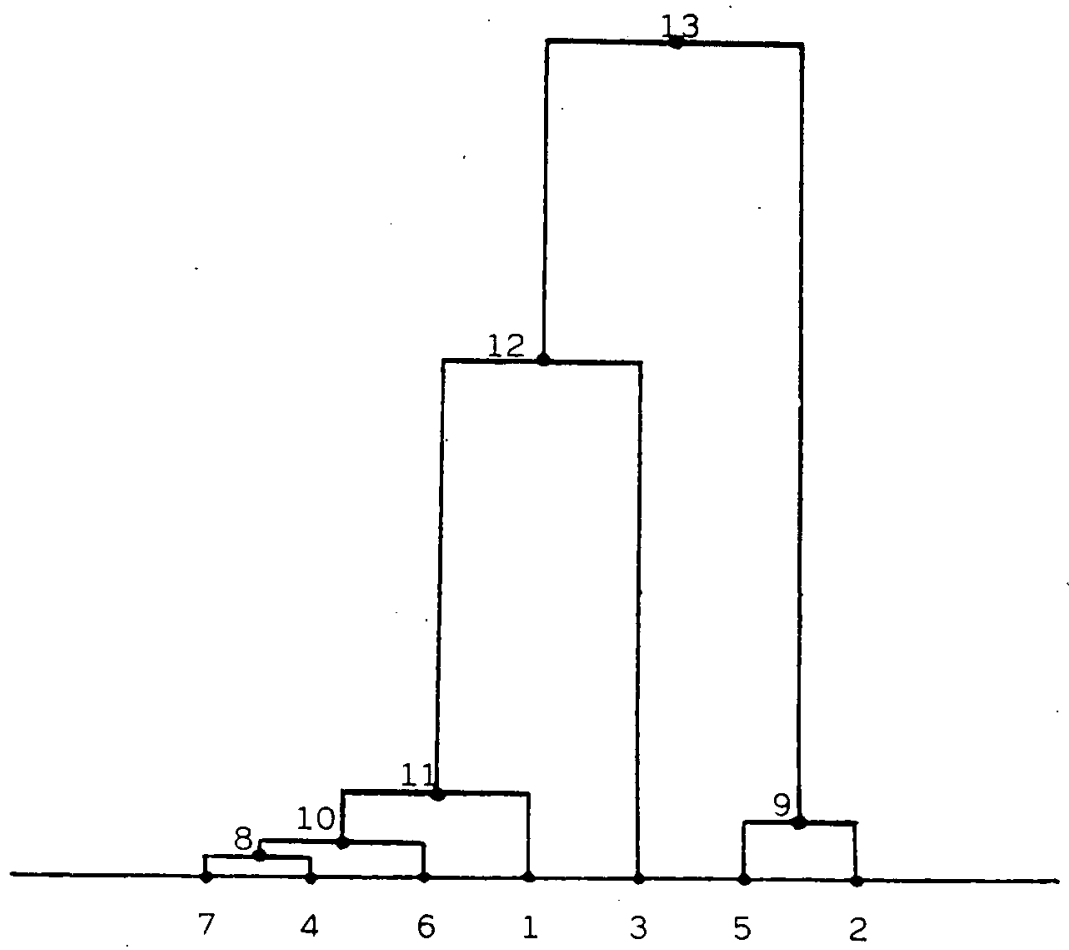

Dendrograma que representa las sucesivas agregaciones entre las clases

Los Cuadros, 8, 9 y 10 recogen, respectivamente, la edición de ordenador de las clases 1,2 y 3 . Para cada clase aparece en la primera franja horizontal el número de la clase, los efectivos que la componen y el porcentaje que tales efec- 
tivos representan en la muestra total. Las columnas 1 y 4 contienen, respectivamente, las etiquetas de la pregunta y de la modalidad de respuesta. Las columnas 2 y 3 recogen la pregunta y la modalidad de respuesta. La columna CRITERIO contiene los valores criterios de cada modalidad, ordenados, como se ve (columna PROBA) por orden de significatividad de la modalidad en la clase. La columna PESO recoge el número de individuos del total de la muestra que han elegido esa modalidad, dato que es recogido en términos de porcentaje en la columna PORCENTAJE GLOBAL. La columna PORCENTAJE MOD/CLA muestra el porcentaje de sujetos de la clase que han elegido o están en esa modalidad de respuesta. Finalmente, la columna PORCENTAJE CLA/MOD muestra qué porcentaje de los sujetos que han elegido esa modalidad de respuesta o pertenecen a ella son miembros de la clase de que se trate. Esta columna equivale a los porcentajes por filas, mientras que la anterior contiene los porcentajes por columnas.

En lo que a la relación de las clases con los factores previamente analizados se refiere, las clases 2 y 3 se definen sobre todo por su importancia en el factor 1, la clase 2 en el espacio que está por encima de la zona central y la clase 3 en el espacio que está por debajo. Por su parte, la clase 1 está definida por su posición en las modalidades de respuesta del factor 4 a que nos referimos más arriba. Las 30 modalidades de respuesta que describen cada clase incluyen, en todo o en gran parte, las 15 modalidades correspondientes a la zona del factor correspondiente en cada caso. Por razones expositivas, la breve descripción de cada clase que viene a continuación se hace de acuerdo con el siguiente orden: clase 2 , clase 3 y clase 1 .

Como se ve en el Cuadro 9, los integrantes de la clase 2 son personas que mayoritariamente ( $91 \%$ ) tienen un bajo nivel de estudios, con escasa información sobre desarrollo y educación, poco sensibles a las cualidades psicológicas de la interacción con el niño, de ideas innatistas, con un bajo nivel de influencia percibida sobre el desarrollo y que defienden prácticas de educación de naturaleza coercitiva. En un $67 \%$ de los casos, se trata de personas de procedencia rural. Se trata de padres tradicionales.

Los hombres y las mujeres integrantes de la clase 3 (ver Cuadro 10) tienen en general un elevado nivel educativo ( $87 \%$ ), son con mayor frecuencia de procedencia urbana (79\%), defienden la interacción herencia-medio como causante de buena parte de las diferencias interindividuales, parecen poco estereotipados en sus ideas, permisivos, sensibles a las cualidades psicológicas de la interacción con el niño, sus expectativas evolutivas son optimistas y el grado de influencia que creen tener sobre el niño es elevado. Su nivel de información parece ser alto y compartido por el cónyuge. Son padres modernos o actualizados.

Finalmente, las personas que integran la clase 1 (Cuadro 8 ), que proceden en un $47 \%$ del nivel educativo medio (y, como luego veremos, en un $48 \%$ del nivel bajo), presentan un nivel de búsqueda de información alto, aunque sea muy bajo el nivel de información al parecer retenida. Son ambientalistas, pero parecen percibir que el control del ambiente educativo se les escapa. Tienen positivas expectativas evolutivas, pero no parece que de ellas deriven prácticas interactivas en consonancia con esa precocidad. Presentan ideas a veces muy poco y a veces muy esterotipadas. Por todo ello, más que intermedia, la posición de este grupo parece oscilante entre las clases 2 y 3 de manera alternativa. Una buena parte de las personas de este grupo $(62 \%)$ tienen experiencia previa como padres. Son padres paradójicos.

El último paso en nuestro programa de análisis de los datos consiste en determinar a cuál de las 6 clases constituidas se asigna a cada uno de los 278 sujetos de nuestra muestra. Saber a qué clase pertenece cada sujeto nos permite 


\begin{tabular}{|c|c|c|c|c|c|c|c|c|}
\hline & & & & & \multicolumn{4}{|c|}{ PORCENTAJES } \\
\hline & CLASE 1 & & $\left(* 1^{*}\right)$ & $\begin{array}{c}\text { CRITERIO } \\
\text { PROBA }\end{array}$ & $\begin{array}{c}\text { PESO } \\
94\end{array}$ & $\begin{array}{c}\text { GLOBAL } \\
33.8\end{array}$ & MOD/CLA & CLA/MOD \\
\hline NEPP & Nivel de estudios de la pareja (tomado del padre) & $=$ Medio & (NEP2) & 7.310 .000 & 98 & 35.3 & 64.9 & 62.2 \\
\hline PROI & Si se ha informado, ¿ por que procedimiento? & $=$ Leído libros & (PRO2) & 5.590 .000 & 87 & 31.3 & 53.2 & 57.5 \\
\hline MAFE & ¿Existe relación entre cómo se encuentran madre y niño? & $=S_{i}$ & (MAF1) & 5.490 .000 & 257 & 92.4 & 100.0 & 36.6 \\
\hline LECT & ¿Ha leído alguna cosa sobre niños, cómo son y cómo educarlos? & $=\mathrm{Si}_{\mathbf{i}}$ & (LEC1) & 4.220 .000 & 174 & 62.6 & 78.7 & 42.5 \\
\hline PFOT & Qué le preocupa más del futuro de sus hijos? & = Situación social gen. & (PFO17 & 3.920 .000 & 158 & 56.8 & 72.3 & 43.0 \\
\hline PICA & iA que edad cree que pueden controlar el pipí y la caca? & $=$ Antes 18 meses & (PCI1) & 3.870 .000 & 63 & 22.7 & 36.2 & 54.0 \\
\hline PROF & Profesiôn & $=$ Administrativos & (PRW5) & 3.430 .000 & 29 & 10.4 & 19.1 & 62.1 \\
\hline INEM & Se ha informado de lo que es un embarazo, criar, educ. niños & $=S_{i}$ & (INE1) & 3.400 .000 & 208 & 74.8 & 86.2 & 38.9 \\
\hline COIN & Si cree que puede influir sobre intelig., icómo pude hacerlo? & $=$ Ejemplo & (COI2) & 3.380 .000 & 50 & 18.0 & 28.7 & 54.0 \\
\hline APLE & Recuerda alguna cosa concreta que hava leído? & $=$ Nada o impreciso & (APL2) & 3.220 .001 & 101 & 36.3 & 48.9 & 45.5 \\
\hline LEPM & Si el niño empieza a llorar de noche zquién debe levantarse? & $=$ Padre & (LEP1) & 3.210 .001 & 12 & 4.3 & 9.6 & 75.0 \\
\hline CITI & Si cree que se puede ayudar timidez, ¿qué tipo de cosas? & $=$ Potenciar relaciones & (CIT2) & 3.120 .001 & 40 & 14.4 & 23.4 & 55.0 \\
\hline DPBE & Hay niños alegres-serios, lanzados-tímidos. ¿A qué se debe? & = Educación & (DPB2) & 2.990 .001 & 71 & 25.5 & 36.2 & 47.9 \\
\hline CILE & Qué cosas se pueden hacer estimular lenguaje? & $=$ Hablar, estimular & (CIL1) & 2.880 .002 & 164 & 59.0 & 70.2 & 40.2 \\
\hline IDES & ¿Hasta dónde le gustaría que llegara su hijo en estudios? & $=$ Universidad & (IDE2) & $2.84 \quad 0.002$ & 134 & 48.2 & 59.6 & 41.8 \\
\hline PAII & Paridad 2 & $=$ No primípara & (PAI2) & 2.840 .002 & 140 & 50.4 & 61.7 & 41.4 \\
\hline EDSX & ¿A partir de qué edad cree que son distintos? & $=$ NO CONTESTA & (EDS7) & 2.790 .003 & 40 & 14.4 & 22.3 & 52.5 \\
\hline EXPR & Qué profesión cree que realmente tendrá? & $=$ Imposible saber & (EXP7) & 2.730 .003 & 63 & 22.7 & 31.9 & 47.6 \\
\hline PQHU & ¿Por qué es malo ser hijo único? & $=$ Unico, mimado & (PQH2) & 2.640 .004 & 113 & 40.6 & 51.1 & 42.5 \\
\hline EERR & A partir de qué edad empezar a explicar y razonar cosas? & $=2-4$ años & (EER4) & 2.640 .004 & 77 & 27.7 & 37.2 & 45.5 \\
\hline $\mathrm{CICO}$ & Qué tipo de cosas pueden hacer niño vaya bien colegio? & $=$ Colaborar profesores & (CIC4) & 2.620 .004 & 14 & 5.0 & 9.6 & 64.3 \\
\hline CPRO & De qué depende que tengan una profesión mejor que otra? & $=$ Incontrolados & (CPR5) & 2.620 .004 & 34 & 12.2 & 19.1 & 52.9 \\
\hline EHIM & Edad del hijo mayor & $=3-4$ & $(\mathrm{EHI} 2)$ & 2.620 .004 & 64 & 23.0 & 31.9 & 46.9 \\
\hline PRPC & Niño suyo se hiciera pipi-caca $4-5$ años. Se preocuparía? & $=$ Un poco & (PRP3) & 2.560 .005 & 32 & 11.5 & 18.1 & 53.1 \\
\hline GUMI & Le gustaría saber más cosas sobre los niños? Desarr. y educ. & $=\mathrm{Si}_{\mathrm{i}} \mathrm{p}$ & (GUM1) & 2.550 .005 & 247 & 88.8 & 94.7 & 36.0 \\
\hline PARI & Paridad 1 & $=$ Segundípara & (PAR2) & 2.540 .006 & 100 & 36.0 & 45.7 & 43.0 \\
\hline ISEO & Un de 11 años piensa igual que uno de 6 , la misma inteligencia? & $=S_{i}$ & (ISE1) & 2.530 .006 & 37 & 13.3 & 20.2 & 51.4 \\
\hline
\end{tabular}


Cundro 9

Modalidades de respuesta que describen a la clase 2

\begin{tabular}{|c|c|c|c|c|c|c|c|c|}
\hline & & & & & \multicolumn{4}{|c|}{ PORCENTAJES } \\
\hline & CLASE 2 & & $\left(* 2^{*}\right)$ & $\begin{array}{c}\text { CRITERIO } \\
\text { PROBA }\end{array}$ & $\begin{array}{c}\text { PESO } \\
79\end{array}$ & $\begin{array}{c}\text { GLOBAL } \\
28.4\end{array}$ & MOD/CLA & CLA/MOD \\
\hline NEPP & Nivel de estudios de la pareja (tomado del padre) & $=$ Bajo & (NEP1) & 9.580 .000 & 104 & 37.4 & 82.3 & 62.5 \\
\hline NESS & Nivel de estudios & $=\mathrm{Bajo}$ & (NES1) & 9.560 .000 & 134 & 48.2 & 91.1 & 53.7 \\
\hline QLEC & En caso afirmativo ¿qué ha leído? & $=$ NO CONTESTA & (QLE6) & 7.950 .000 & 101 & 36.3 & 73.4 & 57.4 \\
\hline APLE & ¿Recuerda alguna cosa concreta que haya leído? & $=$ NO CONTESTA & (APLE3) & 7.720 .000 & 104 & 37.4 & 73.4 & 55.8 \\
\hline LECT & ¿Ha leído alguna cosa sobre niños, cómo son y cómo educarlos? & $=$ No & (LEC2) & 7.030 .000 & 93 & 33.5 & 65.8 & 55.9 \\
\hline INEM & ¿Se ha informado de lo que es un embarazo, criar, educ. niños? & $=\mathrm{No}$ & (INE2) & 6.740 .000 & 70 & 25.2 & 54.4 & 61.4 \\
\hline IANT & Si los antojos influyen, ¿en qué aspectos? & $=$ Físicos & (IAN1) & 6.540 .000 & 34 & 12.2 & 34.2 & 79.4 \\
\hline JUGE & Si ve a su hijo-hija jugando con sus genit. ¿Qué haría? & $=$ Reñir, castigar & (JUG2) & 6.470 .000 & 45 & 16.2 & 40.5 & 71.1 \\
\hline ANTE & Si una embarazada tiene un antojo y no puede satisfacer, jinfluye? & $=\mathrm{Si}_{\mathrm{i}}$ & (ANT1) & 5.949 .999 & 49 & 17.6 & 40.5 & 65.3 \\
\hline EERR & ¿A partir de qué edad empezar a explicar y razonar cosas? & $=$ Más 4 años & (EER5) & 5.770 .000 & 58 & 20.9 & 44.3 & 60.3 \\
\hline PROI & Si se ha informado, ¿por qué procedimientos? & $=$ Otras combinaciones & (PRO9) & 5.610 .000 & 85 & 30.6 & 55.7 & 51.8 \\
\hline ESJU & Si ve hijo 4-5 años juega con muñecas, hija con pistola & = Quitárselos & (ESJ3) & 5.430 .000 & 41 & 14.7 & 34.2 & 65.9 \\
\hline PQIE & ¿Por qué levantarse madre de noche? & $=$ Madre sabe tratar & (PQL1) & 5.350 .000 & 54 & 19.4 & 40.5 & 59.3 \\
\hline BETI & Qué se puede hacer con un niño 1 año que tira cosas & $=$ Reñir castigar & (BET2) & 5.200 .000 & 38 & 13.7 & 31.6 & 65.8 \\
\hline HCON & ¿Ha hablado mucho de estas cosas con su cónyuge? & $=\mathrm{Nada}$ & (HCO4) & 5.180 .000 & 16 & 5.8 & 16.7 & 87.5 \\
\hline ELPM & Si el niño empieza a llorar de noche Quién debe levantarse? & $=$ Madre & (LEP2) & 5.030 .000 & 65 & 23.4 & 44.3 & 53.8 \\
\hline BENE & ¿Qué es bueno que haga la embarazada para beneficiar niño? & $=$ Cuidados físicos & (BEN1) & 4.780 .000 & 185 & 66.5 & 86.1 & 36.8 \\
\hline HABI & Hábitat & $=$ Rural & (HAB1) & 4.720 .000 & 126 & 45.3 & 67.1 & 42.1 \\
\hline EREN & ¿A partir de qué edad hay que empezar a reñir? & $=5$ años o más & (ERE3) & 4.620 .000 & 11 & 4.0 & 12.7 & 90.9 \\
\hline AZUR & Si su hijo fuera zurdo, ¿qué haría? & $=$ Tratar de cambiar & (AZU2) & 4.570 .000 & 53 & 19.1 & 36.7 & 54.7 \\
\hline BOCA & ¿Por qué cree que los niños se lo llevan todo a la boca? & $=$ Molestias dientes & (BOC3) & 4.560 .000 & 33 & 11.9 & 26.6 & 63.6 \\
\hline ESTX & ¿L gustaría que su hijo fuera muy mascu. hija muy fem.? & $=\mathrm{S}_{\mathbf{1}}$ & (EST1) & 4.540 .000 & 111 & 39.9 & 60.8 & 43.2 \\
\hline FEOY & ¿Cree ud. que el feto oye? & $=\mathrm{No}$ & (EFO2) & 4.420 .000 & 49 & 17.6 & 34.2 & 55.1 \\
\hline ORID & ¿De dónde cree ha sacado ideas sobre los niños y la edu.? & $=$ Experiencia previa & (ORII) & $4.35 \quad 0.000$ & 110 & 39.6 & 59.5 & 42.7 \\
\hline ITIM & Niño 4.5 años muy tímido. ¿Pueden hacer algo los padres? & $=\mathrm{No}$ & (ITI2) & 4.320 .000 & 42 & 15.1 & 30.4 & 57.1 \\
\hline BQPP & ¿Por qué padre presenciar parto? & $=$ Evitar nervios & (PQP1) & 4.260 .000 & 40 & 14.4 & 29.1 & 57.5 \\
\hline DLEN & ¿Por qué unos niños hablan mejor y otros peor? & $=$ Herencia & (DLE1) & 4.170 .000 & 77 & 27.7 & 45.6 & 46.8 \\
\hline QMFE & Si hay relación madre feto zen qué cosas existe? & $=$ NO CONTESTA & (QMF5). & 4.110 .000 & 20 & 7.2 & 17.7 & 70.0 \\
\hline CILE & ¿Qué cosas se pueden hacer estimular lenguaje? & $=$ NO CONTESTA & (CIL5) & 4.010 .000 & 61 & 21.9 & 38.0 & 49.2 \\
\hline FCOG & ¿Es importante coger niños con frecuencia o al contrario? & $=$ Menos posible & $(\mathrm{FCO} 2)$ & 3.960 .000 & 151 & 54.3 & 72.2 & 37.7 \\
\hline
\end{tabular}




\begin{tabular}{|c|c|c|c|c|c|c|c|c|}
\hline & & & & & \multicolumn{4}{|c|}{ PORCENTAJES } \\
\hline & CLASE 3 & & $\left(* 3^{*}\right)$ & $\begin{array}{c}\text { CRITERIO } \\
\text { PROBA }\end{array}$ & $\begin{array}{c}\text { PESO } \\
67\end{array}$ & $\begin{array}{c}\text { GLOBAL } \\
24.1\end{array}$ & MOD/CLA & CLA/MOD \\
\hline NESS & Nivel de estudios & $=$ Alto & (NES3) & 11.640 .000 & 72 & 25.9 & 86.6 & 80.6 \\
\hline NEPP & Nivel de estudios de la pareja (tomado del padre) & $=$ Alto & (NEP3) & 11.330 .000 & 76 & 27.3 & 86.6 & 76.3 \\
\hline DINT & ¿A qué se debe niños normales unos sean más inteligentes? & $=$ Herencia-educación & (DIN3) & $8.70 \quad 0.000$ & 60 & 21.6 & 64.2 & 71.7 \\
\hline PROF & Profesión & $=$ Técnicos superiores & (PRW7) & 8.660 .000 & 32 & 11.5 & 44.8 & 93.8 \\
\hline DPBE & Hay niños alegres-serios, lanzados-tímidos. ¿A qué se debe? & $=$ Herencia-educación & (DPB3) & 7.080 .000 & 51 & 18.3 & 50.7 & 66.7 \\
\hline FAIN & ¿Los padres pueden favorecer la inteligencia de sus hijos? & $=$ Sí $_{1}$ & (FAI1) & 5.950 .000 & 201 & 72.3 & 95.5 & 31.8 \\
\hline JUGE & Si ve a su hijo-hija jugando con sus genti. ¿Qué haria? & $=$ Nada especial & (JUG1) & 5.400 .000 & 126 & 45.3 & 73.1 & 38.9 \\
\hline ITIM & Niño 4-5 años muy tímido. ¿Pueden hacer algo los padres? & $=S_{i}$ & (ITI1) & 5.200 .000 & 231 & 83.1 & 98.5 & 28.6 \\
\hline BENE & ¿Qué es bueno que haga la embarazada para beneficiar niño? & = Cuidados físico-psic. & (BEN4) & 5.200 .000 & s5 & 19.8 & 43.3 & 52.7 \\
\hline HABI & Hábitat & $=$ Urbano & (HAB2) & 4.910 .000 & 152 & 54.7 & 79.1 & 34.9 \\
\hline LECT & ¿Ha leído alguna cosa sobre niños, cómo son y cómo educarlos? & $=\mathrm{Si}_{\mathrm{I}}$ & (LECl) & 4.810 .000 & 174 & 62.6 & 85.1 & 32.8 \\
\hline HCON & ¿Ha hablado mucho de estas cosas con su cónyuge? & $=$ Mucho & (HCO1) & 4.670 .000 & 151 & 54.3 & 77.6 & 34.4 \\
\hline FEPE & ¿Cree que el niño recién nacido ya tiene propia personalidad? & = Sólo en parte & (FEP4) & 4.650 .000 & 24 & 8.6 & 23.9 & 66.7 \\
\hline MAFE & ¿Existe relación entre cómo se encuentra madre y feto? & $=\mathrm{Si}_{1}$ & (MAF1) & 4.650 .000 & 257 & 92.4 & 100.0 & 26.1 \\
\hline ORID & ¿De dónde cree ha sacado ideas sobre los niños y la educación? & $=$ Combinaciones & (ORI4) & $46.40 \quad 0.000$ & 112 & 40.3 & 64.2 & 38.4 \\
\hline AZUR & Si su hijo fuera zurzo, ¿qué haría? & $=$ No intervenir & (AZUR'1) & 4.630 .000 & 213 & 76.6 & 94.0 & 29.6 \\
\hline PFOT & ¿Qué le preocupa más del futuro de sus hijos? & $=$ Felicidad del niño & (PFO2) & 4.210 .000 & 56 & 20.1 & 38.8 & 46.4 \\
\hline IANT & Si los antojos influyen ¿en qué aspectos? & $=$ NO CONTESTA & (IAN4) & 4.190 .000 & 234 & 84.2 & 97.0 & 27.8 \\
\hline EREN & ¿A partir de qué edad hay que empezar a reñir? & $=102$ años & (ERE1) & 4.160 .000 & 136 & 48.9 & 70.1 & 34.6 \\
\hline DLEN & ¿Por qué unos niños hablan mejor y otros peor? & $=$ Medio ambiente & (DLE2) & 4.090 .000 & 104 & 37.4 & 58.2 & 37.5 \\
\hline INEM & ¿Se ha informado de lo que es un embarazo, criar, educ. niños? & $=$ Sí & (INE27 & 4.060 .000 & 208 & 74.8 & 91.0 & 29.3 \\
\hline PERE & ¿Qué debe evitar una embarazada que perjudique al niño? & $=$ Esfuerzos y tensiones & (PER3) & $4.00 \quad 0.000$ & 55 & 19.8 & 37.3 & 45.5 \\
\hline RENF & Si está enfadado con su hijo de 8 años, ¿qué cree que hará? & $=$ Explicar, hablar & (REN1) & 3.950 .000 & 118 & 42.4 & 62.7 & 35.6 \\
\hline MIBE & ¿Qué hacer cuando un niño pequeño tenga miedo? & $=$ Combinaciones & (MIB8) & 3.850 .000 & 19 & 6.8 & 17.9 & 63.2 \\
\hline ESJU & Si ve hijo de 4-5 años juega con muñecas, hija con pistola & $=$ Nada especial & (ESJ1) & 3.840 .000 & 194 & 69.8 & 86.6 & 29.9 \\
\hline ICOL & Los padres pueden hacer algo para que el colegio le baya bien & $=\mathrm{Sí}$ & (ICO1) & 3.840 .000 & 264 & 95.0 & 100.0 & 25.4 \\
\hline LEPM & Si el niño empieza a llorar de noche, ¿quién debe levantarse? & $=$ Depende & (LEP3) & 3.810 .000 & 200 & 71.9 & 88.1 & 29.5 \\
\hline
\end{tabular}


depurar aun más la relación entre las clases y las diferentes variables sociodemográficas.

Así ocurre, por ejemplo, con el nivel de estudios, como muestran los Cuadros 11 y 12. En el Cuadro 11 se recoge la composición de las clases en función del nivel de estudios. Como se ve, la clase 2 (padres tradicionales) está formada en un $91 \%$ por personas de nivel bajo, la clase 3 (padres modernos) en un $87 \%$ por personas de nivel alto y la clase 1 (padres paradójicos) está formada casi paritariamente por personas de los niveles bajo y medio $\left[\mathrm{X}^{2}(10)=232.80\right.$, $p<0.001]$.

Cundro 11

Porcentajes por columnas: composiciōn de las clases en función del nivel de estudios.

\begin{tabular}{|l|c|c|c|c|c|c|}
\hline & 1 & 2 & 3 & 4 & 5 & 6 \\
\hline Bajo & 47.9 & 91.1 & 3.0 & 21.1 & 50.0 & 66.7 \\
\hline Medio & 46.8 & 7.6 & 10.4 & 47.4 & 50.0 & 11.1 \\
\hline Alto & 5.3 & 1.3 & 86.6 & 31.6 & 0 & 22.2 \\
\hline & 100 & 100 & 100 & 100 & 100 & 100 \\
\hline
\end{tabular}

La información del Cuadro 12 es complementaria a la anterior. Recoge los porcentajes por filas, que muestran la distribución porcentual de los efectivos de cada nivel de estudios entre las diferentes clases de padres.

Cundro 12

Porcentajes por filas: distribución porcentual de los efectivos de cada nivel de estudios entre las diferentes clases.

\begin{tabular}{|l|c|c|c|c|c|c|c|}
\hline & 1 & 2 & 3 & 4 & 5 & 6 & \\
\hline Bajo & 33.6 & 53.7 & 1.5 & 3.0 & 3.7 & 4.5 & 100 \\
\hline Medio & 61.1 & 8.3 & 9.7 & 12.5 & 6.9 & 1.4 & 100 \\
\hline Alto & 6.9 & 1.4 & 80.6 & 8.3 & 0 & 2.8 & 100 \\
\hline
\end{tabular}

Como se ve, las personas de nivel de estudios bajo se reparten entre las clases 1 y 2 (padres paradójicos y tradicionales, respectivamente); las de nivel medio están sobre todo en la clase $1 \mathrm{y}$, a una gran distancia, en la 4 (cercana a la 1 en el dendrograma); las personas de nivel alto se concentran claramente en la clase 3 (padres modernos o actualizados).

El examen de la distribución de los hombres y mujeres en las distintas clases (Cuadro 13) muestra que el sexo no guarda relación con la pertenencia a una u otra clase $\left[\mathrm{X}^{2}(5)=6.75, p=0.239\right]$.

\section{Cundro 13}

Distribución porcentual de los hombres y mujeres en cada una de las clases.

\begin{tabular}{|l|c|c|c|c|c|c|}
\hline & 1 & 2 & 3 & 4 & 5 & 6 \\
\hline Mujeres & 51.1 & 45.6 & 44.8 & 68.4 & 50.0 & 77.8 \\
\hline Hombres & 48.9 & 54.4 & 55.2 & 31.6 & 50.0 & 22.2 \\
\hline & 100 & 100 & 100 & 100 & 100 & 100 \\
\hline
\end{tabular}


La experiencia previa como padres tampoco da lugar a diferencias significativas $\left[\mathrm{X}^{2}(10)=12.25, p=0.268\right]$. No obstante, y como muestra el Cuadro 14 , es obvio que en las clases 2 y 3 la paridad juega un papel menor del que juega en la clase 1 (padres paradójicos), donde predominan las personas con experiencia previa.

\section{Cundro 14}

Distribuciōn de las personas en las distintas clases en función de que tengan o no experiencia previa como padres.

\begin{tabular}{|l|c|c|c|c|c|c|}
\hline & 1 & 2 & 3 & 4 & 5 & 6 \\
\hline Primíparas & 38.3 & 57.0 & 52.2 & 52.6 & 70.0 & 55.6 \\
\hline No primiparas & 61.7 & 43.0 & 47.8 & 47.4 & 30.0 & 44.4 \\
\hline & 100 & 100 & 100 & 100 & 100 & 100 \\
\hline
\end{tabular}

Finalmente, la última variable relevante es el hábitat. El Cuadro 15 recoge la distribución en las diferentes clases de las personas rurales y urbanas. Las diferencias son en este caso significativas $\left[\mathrm{X}^{2}(S)=31.38, p<0.001\right]$. Como puede verse, mientras que los de la clase 1 (paradójicos) son con parecida proporción de procedencia rural o urbana, los padres de la clase 2 (tradicionales) proceden sobre todo de entornos rurales, mientras que los de la clase 3 (modernos) proceden sobre todo de entornos urbanos.

Cundro 15

Distribución de los componentes de las distintas clases en función de su procedencia rural o urbana.

\begin{tabular}{|l|c|c|c|c|c|c|}
\hline & 1 & 2 & 3 & 4 & 5 & 6 \\
\hline Rurales & 45.7 & 67.1 & 20.9 & 36.8 & 50.0 & 44.4 \\
\hline Urbanos & 54.3 & 32.9 & 79.1 & 63.2 & 50.0 & 55.6 \\
\hline & 100 & 100 & 100 & 100 & 100 & 100 \\
\hline
\end{tabular}

Un último dato concierne a la concordancia que se da o no se da entre los miembros de cada pareja. Puesto que sabemos a qué clase pertenece cada uno de nuestros sujetos, podemos adivinar si hay o no coincidencia entre ellos en lo que respecta a la pertenencia a una misma clase. Según nuestros datos, la mitad de los sujetos de la clase 1 son cónyuges (48 de 94) y otro tanto ocurre con la clase 2 (de las 79 personas que hay en este grupo, 40 son marido y mujer). La concordancia es mucho mayor en la clase 3, puesto que de las 67 personas que la componen, 48 (el $78 \%$ ) son pareja. Así pues, la concordancia en el interior de cada pareja es muy superior entre los padres modernos o actualizados que entre los padres tradicionales y los paradójicos.

\section{DISCUSION Y CONCIUSIONES}

En aras de una mayor claridad y coherencia expositiva, los contenidos que siguen aparecen ordenados según el mismo esquema que utilizamos en el artícuculo anterior. Así, nos referiremos en primer lugar a la diversidad y estructura de las ideas de los padres, después a los contenidos de tales ideas y finalmente a sus determinantes. 
Utilizaremos entremezclados los datos procedentes de los dos tipos de análisis realizados, que son complementarios los unos de los otros, aunque hay ocasiones en que tal complementariedad sea menos clara por el hecho de que con $\mathrm{X}^{2}$ podemos servirnos de todas las preguntas del CIP, mientras que el análisis de correspondencias múltiples aporta sólo 30 preguntas, con sus respuestas correspondientes, para cada clase.

\subsection{Diversidad y estructura de las ideas de los padres}

Los datos de nuestra investigación han puesto de manifiesto que las ideas de los padres son muy diversas. Ello no obstante, los diferentes contenidos de las ideas evolutivo-educativas de los padres no parecen estar completamente desconectados los unos de los otros. Nuestros datos corroboran en buena medida los de McGillicuddy-DeLisi (1982a) a este respecto: las ideas de los padres pueden agruparse en subsistemas entre los cuales existe un cierto grado de coherencia lógica. En algunos casos, según nuestros datos, estos subsistemas están bastante bien articulados entre sí, mientras que en otras ocasiones no lo están tanto, como también vimos señalar a Sigel (1986). Así, por ejemplo, ocurre que en general los padres ven como más influenciable por el medio el lenguaje de un niño que su inteligencia, que se considera más determinada por la herencia. En coherencia con ello, su influencia percibida es mayor sobre un aspecto que sobre otro. En nuestro caso, los padres de nivel alto de la clase 3 y los de nivel bajo de la clase 2 , son los que, con contenidos de signo frecuentemente opuestos, presentan en el interior de sus respectivas ideas una articulación más clara entre subsistemas. Por su parte, los padres de nivel de estudios medio y los de nivel bajo de la clase 1 (padres paradójicos), son los que presentan más signos de falta de correspondencia.

En parte relacionado con el problema de la estructura de las ideas, pero mucho más puntual, está el hecho de las contradicciones internas entre unas ideas y otras. Tales contradicciones existen y, según nuestros datos, son más frecuentes en los niveles educativos bajo y medio que en el alto.

\subsection{Contenidos de las ideas de los padres}

En relación con las causas determinantes de las características psicológicas y de las diferencias interindividuales, nuestros datos son en general coincidentes con los aportados por investigaciones anteriores. En los dos extremos, los padres de nivel bajo defienden frecuentemente posiciones innatistas, mientras que los de nivel alto defienden la interacción herencia-medio. Coincidimos en esto, por citar sólo a algunos, con Boltanski (1969), Frankel y Roer-Bornstein (1982), Schaefer y Edgerton (1985) y Zukow (en este dossier). Nuestros datos van, sin embargo, un poco más allá e indican la existencia de una posición en cierto modo intermedia ocupada por los padres de nivel medio y los de nivel bajo integrados en la clase 1: ambientalistas convencidos, se sitúan como si el ambiente fuera una influencia que ellos no pueden evitar ni controlar.

En lo que respecta a las ideas de los padres sobre el calendario evolutivo, nuestros datos muestran que en general los padres de nivel educativo alto atribuyen capacidades más precoces al feto, al bebé y al niño, que las que atribuyen los padres de nivel bajo. Las diferencias son estadísticamente significativas sólo en el caso de hitos evolutivos que no son tan populares como ver, oír o andar solos (por ejemplo, mantenerse sentado sin ayuda). En estos casos, las diferencias se dan no sólo en función del nivel de estudios y del ocupacional, sino también 
en función del sexo (mayor precocidad en las mujeres que en los hombres) y del grado de experiencia previa como padres (en ocasiones son las primíparas quienes muestran expectativas de mayor precocidad, mientras que otras veces son las no primíparas). En general, estos datos son coincidentes con los de Goodnow et al. (1984), Hess et al. (1980) y Ninio (1979). Nuestros datos evidencian de nuevo una posición paradójica en los padres de nivel educativo medio: a veces próximos a los de nivel alto y a veces a los de nivel bajo, en otras ocasiones parecen quedarse entre unos y otros. Ello muestra el importante grado en que estos padres participan en la clase 1 (padres paradójicos).

Los datos relacionados con los valores, aspiraciones, expectativas y actitudes de los padres son también coincidentes con los de otros autores. Así, por ejemplo, ocurre con la valoración que los padres hacen de la dependenciaindependencia de sus hijos respecto a ellos: las mujeres, las personas urbanas y los padres de elevado nivel ocupacional y educativo se inclinan con mayor frecuencia por valorar la independencia (Gerris et al., 1986; Johnson y Martin, 1985; Kohn, 1969; Lautrey, 1980; Schaefer y Edgerton, 1985; Segal, 1985; Wright y Wright, 1976). Respecto a la deseabilidad de las conductas sexualmente estereotipadas, han sido los padres de nivel educativo bajo quienes sobre todo han manifestado desear que sus hijos fueran muy masculinos y sus hijas muy femeninas.

En lo referente a las cosas que más preocupan a los padres en relación con la educación y el futuro de sus hijos, nuestros datos apuntan en una dirección similar a los de Goodnow (1981, 1985), según los cuales los padres ven como más importante lo que más problemático les parece, aunque en nuestro caso parecen ver como importante sobre todo aquello que perciben que escapa a su control.

Nuestros datos coinciden plenamente con la teoría de la «elasticidad de los valores» (Rodman, 1963) y con la serosión de aspiracioness a que se refieren Rodman y Voydanoff (1978): cuando se pregunta a los padres hasta dónde les gustaría que llegara su hijo en algún aspecto y luego se les interroga respecto a hasta dónde creen que realmente llegarán, las discrepancias más reducidas entre el deseo y la esperanza se dan en los padres de nivel educativo alto, mientras que las discrepancias más acentuadas se dan entre los de nivel bajo. Los padres de nivel medio ocupan aquí una posición intermedia.

Aún en relación con este tipo de expectativas, no hemos encontrado que difirieran en función del sexo del niño, al igual que ocurrió en la investigación de Intons-Peterson (1985).

Por lo que a las actitudes educativas se refiere, nuestros datos están en la línea de los que muy distintos autores, empezando por Newson y Newson (1963), han puesto de manifiesto: las actitudes coercitivas disminuyen según aumenta el nivel educativo, ocurriendo lo contrario con las permisivas. Los padres de nivel de estudios bajo parecen más orientados en sus prácticas educativas a la formación de hábitos; los de nivel alto aparecen como más razonadores e interactivos; los de nivel medio ocupan aquí una posición que se sitúa entre las dos anteriores.

Los datos relacionados con cómo aprenden los niños y cuál es el papel de los padres, lógicamente, guardan relación con los comentados al principio de este apartado: los padres de nivel educativo alto perciben en sí mismos una capacidad para incidir sobre el desarrollo del niño mucho mayor que la percibida por los padres de nivel bajo; sus ideas suelen implicar una mayor dosis de interaccionismo educativo, mientras en el otro extremo se adoptan posturas que están más próximas a ideas de auto-regulación detivadas de concepciones maduracionistas. Estos datos van en la dirección de los que ya mencionamos para este mismo conjunto de contenidos (Johnson y Martin, 1985; Ninio, 1979; Skinner, 
1985; Tulkin y Cohler, 1973; Zukow, en este dossier). Entre un buen número de los padres de nivel medio hay, como hemos indicado al principio, una concepción ambientalista del aprendizaje, pero el papel de los padres aparece ahí difuminado: es como si percibieran una elevada capacidad del ambiente para influir sobre el niño, pero una capacidad de influencia personal considerablemente menor.

En relación con el papel del padre en el primer año de vida del niño, las mujeres, las personas sin experiencia previa como padres y las de alto nivel de estudios son quienes acentúan más un papel activo del padre, al mismo nivel que la madre. Por otra parte, y refiriéndonos ahora a las cualidades de la relación madre-hijo durante el embarazo, con el aumento del nivel de estudios se observa no sólo una mayor defensa de las interacciones, sino también un énfasis creciente en sus aspectos psicológicos.

\subsection{Determinantes de las ideas de los padres}

Los dos tipos de análisis a que hemos sometido nuestros datos son coincidentes al señalar que el nivel educativo de los padres es el más importante determinante de las diferencias observadas entre unos padres y otros en sus ideas evolutivo-educativas. Por no hacer más larga la lista, coincidimos en esto con Alwin (1984), Boltanski (1969), Bronfenbrenner et al. (1984), Laosa (1982a y b), y Wright y Wright (1976). Y no sólo es el determinante más importante, sino que además lo es con gran diferencia sobre los demás aspectos que influyen sobre las ideas de los padres: cualquiera que sea la variable distinta al nivel de estudios que se tome como criterio, su influencia sobre las ideas de los padres parece estar en todo o en buena parte subordinada al nivel de estudios de los padres. Resulta, sin duda, paradójico que mientras que según muchos críticos la escuela fracasa a la hora de cumplir los objetivos de aprendizaje que se plantea y propone, tenga, por el contrario, tanto éxito en un tema que está muy alejado de sus objetivos educativos. La influencia debe ejercerse a través de la apertura de ideas que la escolarización proporciona, la capacidad de análisis, el espíritu crítico, la disponibilidad para aprender, la mayor capacidad para acceder selectivamente a medios de comunicación y difusión de información, etc.

De todas formas, no existe una conexión mecánica y necesaria entre nivel de estudios e ideas evolutivo-educativas de los padres. Como puede apreciarse en los Cuadros 11 y 12, las ideas más tradicionales y conservadoras (clase 2) son defendidas casi en solitario por un buen porcentaje de padres de nivel educativo bajo (el $54 \%$ de los padres de nivel bajo están en la clase 2, sin apenas compañía de padres de otro nivel, pues representan el $91 \%$ de los padres de dicha clase). Las ideas evolutivo-educativas más actualizadas y más próximas a las de los psicólogos evolutivos y de la educación, son defendidas por un $81 \%$ de los padres de nivel de estudios alto, que representan el $87 \%$ de los padres de esa clase. Sin embargo, el tipo de ideas más oscilante, paradójico y a veces errático es defendido por el $34 \%$ de los padres de nivel bajo y el $61 \%$ de los padres de nivel medio, que aportan, respectivamente, el $48 \%$ y el $47 \%$ de los miembros de la clase 1.

Por lo que a otras variables socio-demográficas se refiere, el papel del sexo y el hábitat, allî donde es importante, aparece subordinado al del nivel de estudios, mientras que en lo relativo al papel del status ocupacional nos sentimos más próximos a Wright y Wright (1976) que a Kohn (1969): el papel determinante jugado por el nivel de estudios es más importante que el jugado por el nivel ocupacional. 
Naturalmente, y como ya señalamos, las fuentes de influencia no están aisladas unas de otras y actúan acumulativamente. La variable nivel ocupacional juega, sin duda, un cierto papel; así, viendo la percepción positiva que tienen los padres de la clase 3 de su capacidad de influencia sobre el niño y su desarrollo, no se puede dejar de pensar en la función directiva que ejercen en la sociedad los detentadores del conocimiento y la cultura; viendo la negativa percepción de la capacidad de influencia personal que presentan los padres de la clase 2 , es difícil no pensar en el escaso control que tal vez tengan sobre sus propias biografias las personas que componen esta clase; los padres de la clase 1, que tal vez ocupen posiciones sociales intermedias, saben que el control es posible, pero saben también que no está en sus manos. No obstante, y aun reconociendo el papel singular jugado por la variable status ocupacional, creemos con Wright y Wright (1976) que cuando dicha variable no esté en consonancia con el nivel de estudios, éste resulta más determinante.

En relación con la experiencia previa como padres, no parece jugar un papel importante entre los padres de las clases 2 y 3 , mientras que sí lo juega entre los de la clase 1 . Este hecho se presta, a nuestro entender, a una interpretación tentativa. Según creemos, las ideas evolutivo-educativas de los padres de la clase 2 proceden sobre todo de un trasfondo cultural tradicional en el que los padres aparecen fuertemente anclados; la experiencia o falta de experiencia como padres no modifica esas ideas. En el caso de los padres de la clase 3, sus ideas evolutivo-educativas parecen proceder de un sistema cognitivo activo, reflexivo, filtrador selectivo de informaciones actualizadas; la experiencia previa o la falta de tal experiencia cambia muy poco las cosas. Los padres de la clase 1 carecen a la vez del anclaje cultural que tienen los de la clase 2 y del sistema de conocimientos y adquisición de conocimientos de los padres de la clase 3; a falta de un referente más estable, la experiencia previa como padres se convierte así en ellos en un elemento configurador de ideas evolutivo-educativas. Pero como la experiencia aporta contenidos muy diversos y resulta, además y en general, menos importante que la percepción que de ella se tiene y la forma en que se evalúa e interpreta, las ideas de los padres de la clase 1 aparecen a veces como paradójicas, oscilantes y contradictorias entre sí, producto, como probablemente sean, de lo que Bugental y Shenum (1984) han calificado como un «putpurri de experiencias erráticas».

Finalmente, la mayor concordancia padre-madre en la clase 3 (padres modernos o actualizados) que en la clase 1 (paradójicos) y en la 2 (tradicionales) sugiere que serán precisamente estos padres de la clase 3 los que organicen entornos y desarrollen interacciones que resulten más beneficiosamente estimulantes para los niños. Y ello fundamentalmente por tres razones: presentan zonas de desarrollo próximo percibido más «anchas», poseen ideas que resaltan la importancia de las interacciones y se ven así mismos como capaces de influir positivamente en el desarrollo de sus hijos. Y además padre y madre suelen coincidir en todo lo anterior.

En todo caso, nuestros datos no concuerdan por completo ni con los de quienes han encontrado concordancia en las ideas de marido y mujer (Bronfenbrenner et al., 1984; Triana y Rodrigo, 1985), ni con los de quienes no la han encontrado (Bacon y Ashmore, 1985, 1985; Bates y Bayles, 1984), sino que muestran que el problema de la concordancia debe plantearse diferencialmente en función del tipo de padres considerados.

Queda para investigaciones posteriores (algunas de ellas ya en curso, como se muestra más adelante en este mismo dossier) la tarea de indagar longitudinalmente la evolución de las ideas que aquí hemos estudiado, la forma en que los padres estructuran el entorno educativo familiar y desarrollan las interaccio- 
nes con sus hijos, y el grado en que las diferencias en estos aspectos se traducen en perfiles de competencia y desarrollo distintos en los niños. Queda también para otras investigaciones la indagación de si las clases de ideas que aquí hemos detectado son discernibles ya en la adolescencia o incluso antes, para saber cuándo toman cuerpo las ideas de que aquí hemos estado hablando. Queda como reto aún mayor la tarea de articular intervenciones destinadas a modificar los conjuntos de ideas cuya presencia puede ser considerada un obstáculo para la estimulación y la promoción del desarrollo. Es en estos diferentes ámbitos donde se concentran nuestros esfuerzos actuales.

\section{Resumen}

Se han estudiado las ideas de los padres sobre el desarrollo y la educación de los niños con un cuestionario contestado por 278 sujetos (maridos y mujeres) de diferentes niveles educativos, con diferentes niveles de escolarización, de diferentes häbitats y diverso grado de experiencia previa como padres. Se han encontrado diferencias entre unos sujetos y otros en relación con su nivel de información, sus expectativas evolutivas, su capacidad de influencia percibida sobre el niño y su desarrollo, etc. El nivel de escolarización del sujeto bá resultado ser el más importante determinante de estas diferencias. El análisis de los datos ba puesto en evidencia la existencia de tres clases de padres a las que se propone denominar tradicionales, modernos o actualizados y paradóficos.

\section{Summary}

Parental ideas about children's development and education have been explored through a questionnaire answered by 278 subjects (busbands and wives) with different levels of schooling, from different habitats as well as differen levels of previous experience as parents. Differences between subjects bave been found regarding information level, developmental expectations, perceived capacity of influence on child development, and so on. The subject's level of schooling has been found to be the most important determinant of these differences. Data analysis has evidenced the existence of three different classes of parents that can be designated as traditional, modem or updated and paradoxical.

\section{Referencias}

ALWIN, D. F. (1984). Trends in parental socialization values: Detroit, 1958-1983. American Joumal of Sociology, 90, 359-382.

ApPIEGATE, J. L., Burke, J. A., Burieson, B. R. y Deila, J. G. (1985). Reflection-enhancing parental communication. En I. E. Sigel (Ed.), Parental belief systems. The psychological consequences for children. Hillsdale, N. J.: L. Erlbaum.

ASHMORE, R. D. y BRODZINSKY, D. M. (Eds.) (1986). Thinking about the family: Views of parents and children. Hillsdale, N. J.: Erlbaum.

BACON, M. K. y ASHMORE, R. D. (1985). How mothers and fathers categorize descriptions of social behavior attributed to daughters and sons. Social Cognition, 3, 193:217.

BACON, M. K. Y ASHMORE, R. D. (1986). A consideration of the cognitive activities of parents and their role in the socialization process. En M. K. Bacon y R. D. Ashomore (Eds.). Thinking about the family: Views of parents and children. Hillsdale, N. J.: Erlbaum.

BAIDWIN, A. (1967). Theories of child development. New York: John Wiley.

BARnetr, R. C. (1981). Parental sex-roles attitudes and child-rearing values. Sex Roles, 7, 837-846.

BATES, J. E. y BAYLES, K. (1984). Objetive and subjetive components in mothers' perception of their children from age 6 months to 3 years. Merril-Palmer Quartely, 30, 111-130.

BAUCH, P. A. (1984). The impact of teachers' instructional beliefs on their teaching: implications for research and practice. Trabajo presentado en la reunión anual de la American Educational Research Association, Nueva Orleans.

BeII, R. (1979). Parent, child and reciprocal influences. American Psychologist, 34, 821-826.

BoLransku, L. (1969). Prime éducation et morale de classe. París: Mouton, (Trad. cast.: Puericultura y moral de clase. Barcelona: Laia, 1974).

BRONFENBRENNER, U., AlVAREZ, W. F. y HENDERSON, Ch. R. (1984). Working and watching: maternal employment status and parents' perceptions of their three-year-old-children. Child Development, 55, 1.362-1.378.

BROOKs-GUNN, J. (1986). Maternal beliefs about children's sex-typed characteristics as they relate to maternal behavior. En I. E. Sigel (Ed.), Parental belief systems. The psychological consequences for children. Hillsdale, N. J.: L. Erlbaum.

BROUSSARD, E. R. y HARTNER, M. S. S. (1971). Further consideration regarding maternal perception of the first born. En J. Hellmuth (Ed.). Exceptional infant: Studies in abnormalities. (Vol. 2). New York: Brunner/Mazel.

Bryan, L. R., Coleman, M., Ganong, L. H. y Bryan, S. H. (1986). Person perception: family structure as a cue for stereotyping. Joumal of Marriage and the Family, 48, 169-174.

BugENTAL, D. B. y SHENNUM, W. A. (1984). «Difficulto children as elicitors and targets of adult communication patterns: an attributional-behavioral transactional analysis. Monographs of the Society for Research in Child Development, 49(1), número de seric 205. 
BuRns, A., Homer. R. y Goodnow. J. (1984). Conditions of life and parental values. Australian Joumal of Psycho$\log y, 36,219-237$.

CANTOR, N. y KIHLSTROM, J. F. (1987). Personality and social intelligence. Englewood Cliffs, N. J.: Prentice-Hall. CASHMORE, J. A. y GOODNOW, J. J. (1985). Agreement between generations: A two-process approach. Child Development, 56, 493-501.

Clark, C. M. y PETERSON, P. (1986). Teachers' thought processes. En M. C. Wittrock (Ed.). Handbook of research on teaching. New York: McMillan.

ClaRkE-STEWART, K. A. (1973). Interactions between mothers and their young children: characteristics and consequences. Monographs of the Society for Research in Child Development, 38(6-7), $\mathrm{n}^{\circ}$ de serie 153.

CROCKER. J., FISKE, S. T. y TAYLOR, S. E. (1984). Schematic bases of belief change. En J. R. Eiser (Ed.). Attitudinal judgment. New York: Springer Verlag.

DEVRIES, M. y SAMEROFF. A. (1984). Culture and temperament: influences on infant temperament in three East African societies. American Joumal of Orthopsychiatry, 54, 83-96.

Dix. Th. H. y GruSEC, J. E. (1985). Parent attribution processes in the socialization of children. En I. E. Sigel (Ed.), Parental belief systems. The psychological consequences for children. Hillsdale. N. J.: L. Erlbaum.

Dix. T., Ruble, D. N., GruSEC, J. E. y NIXON, S. (1986). Social cognition in parents: inferential and affective reactions to children of three age levels. Child Development, 57, 879-894.

Donovan, W. L., LeavitT, L. A. y Baluing, I. D. (1978). Maternal physiological responses to infant signals. Psychophysiology, 15, 68-74.

ELIAS. M. J. y UBRIACO. M. (1986). Linking parental beliefs to children's social competence: toward a cognitivebehavioral assessment model. En R. D. Ashomore y D. M. Brodzinsky (Eds.), Thinking about the family: Views of parents and children. Hillsdale, N. J.: Erlbaum.

EMMERICH, W. (1969). The parental role: a cognitive-functional approach. Monographs of the Society for Research in Child Development, $34, \mathrm{n}^{\circ} 8$.

ENGFER, A. y GAVRANIDOU, M. (1984). Sources of stress and support. Their impact on the mother-child relationsbip. Trabajo presentado en la Conferencia Inaugural Europea sobre Psicología Evolutiva: Desarrollo individual y bienestar humano. Groningen, Holanda.

FishBEIN, M. (1980). A theory of reasoned action: some applications and implications. En H. E. Howe y M. M. Page (Eds.), Nebraska Symposium on Motivation, 1979. Lincoln: University of Nebraska Press.

FRANKEL, D. G. y ROER-BORNSTEIN, D. (1982). Traditional and modern contributions to changing infant-rearing ideologies of two ethnic communities. Monographs of the Society for Reseanch in Chil Development, $47\left(\mathrm{n}^{\circ} 196\right)$.

FreEbERG. N. E. y PAYNE, D. T. (1967). Parental influence on cognitive development in early childhood: A review. Child Development, 38, 65-87.

FRY, P. S. (1985). Relations between teenagers' age, knowledge, expectations and maternal behaviour. British Joumal of Developmental Psychology, 3, 47-55.

GerRIS, J. R. M., VermULST, A. A., FrANKEN, W. M. y JANSSENS, J. M. A.M. (1986). Sacial class and parental situation perceptions as determinants of parental value orientations and behaviors. Trabajo presentado en la Segunda Conferencia Europea sobre Psicología Evolutiva. Roma.

GoodNow, J. J. (1981). Everyday ideas about cognitive development. En J. P. Forgas (Ed.), Social cognition. Perspectives on everyday understanding. New York: Academic Press.

GoodNow, J. J. (1985). Change and variation in ideas about childhood and parenting. En I. E. Sigel (Ed.). Parental belief systems. The psychological consequences for children. Hillsdale, N. J.: L. Erlbaum.

GoODNOW. J. J. (1987). Blending developmental and social approaches to adult social cognition: The case of parents' ideas, actions and feelings. Trabajo presentado en la reunión bienal de la Society for Research in Child Development, Baltimore, Maryland.

GOODNOW. J. J., CASHMORE. J., COTTON, S. y KNIGHT, R. (1984). Mothers' developmental timetables in two cultural groups. Intemational Joumal of Psychology, 19, 193-205.

GOODNOW, J. J., KNIGHT, R. y CASHMORE, J. (1986). Adult social cognition: implications of parents' ideas for approaches to development. En M. Perlmutter (Ed.), Minnesota symposia on child development, (Vol. 18). Hillsdale, N. J.: Erlbaum.

HESS, R. D. (1981). Approaches to the measurement and interpretation of parent-child interaction. En R. W. Henderson (Ed.), Parent-child interaction. New York: Academic Press.

Hess, R. D. y MCDEVITT. T. M. (1986). Some antecedents of maternal attributions about children's performance in Mathematics. En R. D. Ashomore y D. M. Brodzinsky (Eds.), Thinking about the family: Views of parents and children. Hillsdale, N. J.: Erlbaum.

Hess, R., Kashigawa, K., Azuma, H., Price, G. G. y Dickson, W. (1980). Maternal expectations for early mastery of developmental tasks and cognitive and social competence of preschool children in Japan and in the United States. International Joumal of Psychology, 15, 259-271.

Ho. D. Y. F. y KANG, T. K. (1984). Intergenerational comparisons of child-rearing attitudes and practices in HongKong. Developmental Psychology, 20, 1.004-1.016.

HoLOW/AY, S. D. y HESS. R. D. (1985). Mothers' and teachers' attributions about children's mathematics performance. En I. E. Sigel (Ed.), Parental belief systems. The psycologicl consequences for children. Hillsdale, N. J.: L. Erlbaum.

HuNT, M. McV. y Paraskevopoulos. J. (1980). CHildren's psychological development as a function of the inaccuracy of their mother's knowledge of their abilities. Joumal of Genetic Psychology, 136, 285-298.

INTONS-PETERSON. M. J. (1985). Fathers expectations and aspirations for their children. Sex Roles, 12, 877.895.

IPSA. J. M., GRAY, M. M. y THORNBURG, K. R. (1984). Childrearing attitudes of parents in person-oriented and third-oriented occupations: a comparison. The Journal of Psychology, 117, 245-250.

JACOB, T., FAGIN. R., PERRY. J. y VAN DYKE, R. (1975). Social class, child age and parental socialization values. Developmental Psychology, 11, 393.

JOHNSON, J. E. y MARTIN, C. (1985). Parents' beliefs and home learning environments: effects on cognitive development. En I. E. Sigel (Ed.), Parental belief systems. The psychological consequences for children. Hillsdale, N. J.: L. Erlbaum. 
KoHN, M. L. (1969). Class and conformity: a study in values. Homewood, Ill.: Dorsey.

KOHN, M. L. (1976). Social class and parental values: another confirmation of the relationship. Comment on Wright and Wright. American Sociological Review, 41, 538-545.

KoHN, M. L. (1977). Reassessment, 1977. En M. L. Kohn (1977), Class and conformity: a study in values. Chicago, Ill.: Chicago University Press ( $2^{2}$ edición; la $1^{2}$ es de 1969 ).

KOHN, M. L. y SCHOOLER. C. (1969). Class, occupation and orientation. American Sociological Review, 34, 659-678.

KUCZYNSKI, L. (1984). Socialization goals and mother-child interaction: strategies for long-term and short-term compliance. Developméntal Psychology, 20, 1.061-1.073.

LAMB. M. E. y EASTERBROOKS. M. A. (1981). Individual differences in parental sensitivity; origins, components and consequences. En M. E. Lamb y L. R. Sherrod (Eds.), Infant social cognition. Hillsdale, N. J.: Erlbaum.

LAMBERT. W. E., HAmers. J. F. y Frasure-Smith. N. (1979). Child-rearing values: A cross-national study. New York: Praeger.

LANGER. E. J. (1978). Rethinking the role of thought in social interaction. En J. H. Havery, W. I. Ickes y R. F. Kidd (Eds.). New directions in attributions research. (Vol. 2). Hillsdale, N. J.: L. Erlbaum.

LAOSA, L. M. (1982a). Families as facilitators of children's intellectual development at 3 years of age. En L. M. Laosa e I. E. Sigel (Eds.), Families as learning environments for children. New York: Plenum Press.

LAOSA, L. M. (1982b). School, ocupation, culture and family. Joumal of Educational Psychology, 74, 791-827.

LAUTREY, J. (1980). Classe sociale, milieu familial, intelligence. París: P. U. F. (Trad. cast.: Clase social, medio familiar e inteligencia. Madrid: Aprendizaje-Visor, 1985).

Lawton, J. T., SChuler, S. G., FoweII. N. y MADSEN, M. K. (1984). Parents perception of actual and ideal childrearing practices. The Joumal of Genetic Psychology, 145, 77-87.

LE VINE, R. (1974). Parental goals: a cross-cultural view. Teachers College Record, 76, 226-239.

LebarT, L. y Morineau, A. (1984). S. P. A. D. París: CESIA.

Lebart. L., Morineau, A. y Feneion, J. P. (1982). Traitement des données statistiques. París: Dunod.

LUESTER, T. y RHOADES, K. (1987). The relation between parental values and parental behavior: $A$ test of the Kohn bypotesis. Trabajo presentado en la reunión bianual de la Society for Research in Child Development, Baltimore, Maryland.

MANCUSO, J. C. y HANDIN, K. H. (1985). Reprimanding: acting on one's implicit theory of behavior change. En I. E. Sigel (ed.), Parental belief systems. The psychological consequences for children. Hillsdale, N. J.: Erlbaum.

MCBRIDE, A. B. (1985). Differences in women's and men's thinking about parent-child interactions. Research in Nursing and Health, 8, 389-396.

MCGuLCUDDY-DeLISI, A. V. (1982a). Parental beliefs about developmental processes. Human Development, 25, $192-200$.

MCGuUCUDDY-DELISI, A. V. (1982b). The relationship between parents' beliefs about development and family constellation, socioeconomic status, and parents' teaching strategies. En L. M. Laosa e I. E. Sigel (Eds.), Families as learming environments for children. New York: Plenum Press.

MCGWUCUDDY-DELISI, A. V. (1985). The selationship between parental beliefs and children's cognitive level. En I. E. Sigel (ed.), Parental belief systems. The psychological consequences for childre. Hillsdale, N.J.: L. Erlbaum.

MCGILUCUDDY-DELISI, A. V., SIGEI, I. E. y JOHNSON, J. E. (1979). The family as a system of mutual influences: parental beliefs, distancing behaviors and children's representational thinking. En M. Lewis y L. A. Rosenblum (Eds.), The child and its family. New York: Plenum Press.

MIIER, S. A. (1986). Parents' beliefs about their children's cognitive abilities. Developmental Psychology, 22, 276-284.

MuLONES, J. (1978). Relationship between perceived child temperament and maternal behavior. Child Development, 49, 1.255-1.257.

MORAN, J. D. y O'BRIEN, G. (1984). Relationship between parental child-rearing attitudes and preschoolers moral jugments. Psychological Reports, 55, 893.894.

MORGAN, W. R., ALWIN, D. F. y GRIFIN, L. J. (1979). Social origins, parental values and the transmission of inequality. American Joumal of Sociology, 85, 156-166.

MoscovicI, S. (1984). The phenomenon of social representations. En R. M. Farr y S. Moscovici (Eds.), Social representations. Cambridge, Ma: Cambridge University Press.

MUNBY, H. (1982). The pace of theachers' beliefs in research on teacher thinking and decision making, and on altemative methodology. Trabajo preparado para la reunión anual de la Southwest Educational Research Association, Austin (Texas).

NEWBERGER, C. M. (1980). The cognitive structure of parenthood: designing a descriptive measure. En R. Selman y R. Yando (Eds.), New directions for child developments, Vol. 7: Clinical development research. San Francisco: Jossey-Bass.

NEwSON, J. y NEwSON, E. (1963). Infant care in a urban community. Londres: Allen \& Unwing. Publicado en 1965 por Penguin Books con el tírulo de Patterns of infant care in an urban community.

NEWSON, J. y NEwSON, E. (1968). Four years old in an urban community. Londres: Allen \& Unwin.

NEWSON. J. y NEwSON. E. (1976). Seven years old in the bome environment. Londres: Allen \& Unwin.

NiNIO, A. (1979). The naive theory of the infant an other maternal attitudes in two subgroups in Israel. Cbild Development, 50, 976-980.

PalACIOS. J. (1984). Las creencias de los padres sobre el desarrollo y la educación de sus bijos. Manuscrito no publicado.

PALACIOS. J. (1985). El papel de las actitudes en el proceso de integración. Trabajo preparado para el Curso de formación sobre el proceso de integración de los niños deficientes en la escuela. Madrid.

PalAcios. J. (1987). Reflexiones en torno a las implicaciones educativas de la obra de Vygotski. En M. Siguán (Coord.), Actualidad de Lev S. Vygotski. Barcelona: Anthropos.

PALACIOS. J. (en prensa). Las ideas de los padres sobre el desarrollo y la educación de sus bijos. Bases para un programa de educación de padres en Andalucía. Sevilla: Instituto de Desarrollo Regional.

13 PALKOVITZ, R. (1984). Parental attitudes and father's interactions with their 5-month-old infants. Developmental Psychology, 26, 1.054-1.060. 
PARKE. R. D. (1978). Parent-infant interaction: progress, paradigms and problems. En G. P. Sackett (Ed.), Observing behavior. Vol. I: Theory and applications in mental retardation. Baltimore, Md: University Park Press.

PARKS, P. L. y SMERGuO. V. L. (1986). Relationships among parenting knowledge, quality of stimulation in the home and infant development. Family Relations, 35, 411-416.

PARSONS, J. E., ADLER. T. F. y KACZALA, C. M. (1982). Socialization of achievement attitudes and beliefs: parental influences. Child Development, 53, 310-321.

Pharis, M. E. y Manosevitz, M. (1980). Parental models: a means for evaluating different parental contexts. En D. B. Sawin, R. C. Hawkins, L. O. Walker y J. H. Penticuff (Eds.), Exceptional infant (Vol. 4). New York: Brunner-Mazel.

Price, G. G. y Guingham, M. G. (1985). Effects of mothers' overestimations and underestimations of their children's intellectual ability: a reanalysis of Hunt and Paraskevopoulos. Joumal of Genetic Psychology, 146, $477-481$.

Rejghart. P. A. (1984). Preservice teacher beliefs about teaching. Trabajo presentado en la reunión anual de la American Educational Research Association, Nueva Orleans.

Roberts, G. C., BLOCK, J. H. y BLOCK. J. (1984). Continuity and change in parent's child-rearing practices. Child Development, 55, 586-597.

Rodman, H. (1963). The lower-class value stretch. Social Forces, 42, 205-215.

Ropman, H. y VoYdanOfF, P. (1978). Social class and parent's range of aspirations for their children. Social Problems, 25, 333-344.

Rodrigo. M. J. (1985). Las teorías implícitas en el conocimiento social. Infancia y Aprendizaje, 31-32, $145-156$. Rogers, C. (1982). A social psycbology of scbooling. The expectancy process. Londres: Rouletge \& Kegan Paul. (Trad. cast. en prensa en la editorial Aprendizaje-Visor).

ROKEACH, M. (1980). Some unresolved issues in theories of beliefs, attitudes and values. En M. M. Page (Ed.), Nebraska Symposium on motivation, 1979: beliefs, attitudes and values. Lincoln: University of Nebraska Press.

Roosi, N. W. (1984). Effects of teenage parenting programs on knowledge and attitudes. Adolescence, 19, 659-666.

Rosenthal, R. y Jacobson, L. (1968). Pygmation in the classroom. New York: Holt, Rinehart \& Winston. (Trad. cast.: Pygmalion en la escuela. Madrid: Marova, 1980).

Ross, L. (1981). The eintuitive scientiso formulation and its developmental implications. En J. H. Flavell y L. Ross (Eds.), Social cognitive development. Frontiers and possible futures. Cambridge: Cambridge University Press.

RUSSEL, A. y RUSSEI, G. (1982). Mother, father and chhild beliefs about child development. Joumal of Psychology, $110,297-306$.

SAMEROFF, A. J. (1975). Early influences on development: fact or fancy? Merrill-Palmer Quarterly, 21, $267-294$.

SAMEROF, A. J. y SeIFER, R. (1983). Familial risk and child competence. Cbild Development, 54, 1.254-1.268.

SAMEROF, A. J. y FeIl, L. A. (1985). Parental concepts of development. En E. I. Sigel (Ed.), Parental belief systems. The psychological consequences for children. Hillsdale, N. J.: L. Erlbaum.

SChAEFER, E. S. y EDGerTon, M. (1985). Parent and child correlates of parental modernity. En I. E. Sigel (Ed.), Parental belief systems. The psychological consequences for children. Hillsdale, N. J.: L. Erlbaum.

SEGAL. M. (1985). A study of maternal beliefs and values within the context of an intervention program. En I. E. Sigel (Ed.), Parental belief systems. The psychological consequences for children. Hillsdale, N.J.: L. Erlbaum.

SEGINER, R. (1983). Parent's educational expectations and children's academic achievements: A literature review. Merrill-Palmer Quarterly, 29, 1-23.

SHAVEISON, R. J. (1983). Review of research on teachers' pedagogical judgments, plans and decisions. The Elementary School Joumal, 83, 392-413.

SIGEL. I. E. (Ed.) (1985a). Parental belief systems. The psychological consequences for children. Hillsdale, N. J.: L. Erlbaum.

SIGEL, I. E. (1985b). A conceptual analysis of beliefs. En I. E. Sigel (Ed.), Parental belief systems. The psychological consequences for children. Hillsdale, N. J.: L. Erlbaum.

SIGEI, I. E. (1986). Reflections on the belief-behavior connection: lessons learned from a research program on parental belief systems and teaching strategies. En R. D. Ashmore y D. M. Brodzinsky (Eds.), Thinking about the family: Views of parents and chbildren. Hillsdale, N. J.: L. Erlbaum.

SIGEL, I. E., MCGILICUdDY-DeLiSI, A.V. y JoHnSON. J. E. (1980). Parental distancing, beliefs and children's representational competence within the family context. Princenton, N. J.: Educational Testing Service.

SKINNER, E. A. (1985). Determinants of mother sensitive and contingent-responsive behavior: The role of childrearing beliefs and socioeconomic status. En I. E. Sigel (Ed.), Parental belief systems. The psychological consequences for children. Hillsdale, N. J.: L. Erlbaum.

SPANGIER, G. (1986). Contemporar and longitudinal relationship between perceived child temperament and quality of parent-child interactions. Trabajo presentado en la Segunda Conferencia Europea sobre Psicología Evolutiva. Roma.

Stern, M. y Hildebrant, K. A. (1986). Permaturity sterotyping: effects on mother-infant interaction. Child Development, $57,308-315$.

STEVENS. J. M. (1984). Black grandmothers and black adolescent mothers' knowledge about parenting. Developmental Psychology, 20, 1.017-1.025.

SuTHERLAND, K. (1983). Parent's belief about child socialization. A study of parenting models. En I. E. Sigel y L. M. Laosa (Eds.), Changing families. New York: Plenum Press.

TACHACHNICK, B. R. y ZEICHNER, K. M. (1985). Individual and contetual influences on the relationships between teacher beliefs and classroom behaviors: case study of two beginning teachers in the United States. Trabajo presentado en la conferencia de la International Study Association on Teacher Thinking, Universidad de Tilburg (Holanda).

Triana, B. y Rodrigo, M. J. (1985). El concepto de infancia en nuestra sociedad: una investigación sobre teorías implícitas de los padres. Infancia y Aprendizaje, 31-32, 157-171.

TULKIN, S. R. y COHIER, B. J. (1973). Childrearing attitudes and mother-child interaction in the first year of life. Merrill-Palmer Quarterly, 19, 95-106.

TURNER, P. H. y HARRIS, M. B. (1984). Parental attitudes and preschool children's social competence. The Journal of Genetic Psychology, 144, 105-113. 
VYGoTSKI, L. S. (1934/1984). Aprendizaje y desarrollo intelectual en la edad escolar. Infancia y Aprendizaje, 27-28, 105-116.

VYGoTSKI, L. S. (1935/1979). Interacción entre aprendizaje y desarrollo. Capítulo 6 de El desarrollo de los procesos psicológicos superiores. Barcelona: Crítica.

WEGNER, D. M. y VAUACHER, R. R. (1977). Implicit psychology: an introduction to social cognition. New York: Oxford University Press.

WHITING, B. B. (1974). Folk wisdom and child-rearing. Merrill-Palmer Quarterly, 20, 9-19.

WRGHT, J. D. y WRIGHT, S. R. (1976). Social class and parental values for children: a partial replication and extension of the Kohn thesis. American Sociological Review, 41, 527-548.

YINGER, R. J. (1986). Investigación sobre el conocimiento y el pensamiento de los profsores. Hacia una concepción de la actividad profesional. Ponencia presentada en la Rábida (Huelva) en un Congreso sobre el pensamiento del profesor.

YONG, K. C. (1981). A comparative study of knowledge and attitudes of child growth and develpment among teenage mothers and adult mothers. Dissertation Abstracts International, 42, 1.474-1475A.

ZeIro, F. A., DunCAN, S. W., BARDEN, R. C., GARBER, J. y MASTERS, J. C. (1986). Adults' expectancies about children's emotional responsivenness: implications for the development of implicit theories of affect. Developmental Psychology, 22, 109-114.

ZUKOW, P. G. (1987). Teorias populares sobre lo que los niños comprenden y prácticas interactivas en una población rural de Méfico Central. En este mismo dossier. 